\title{
Numerical Computation of Instabilities and Internal Waves from In Situ Measurements via the Viscous Taylor-Goldstein Problem
}

\author{
QIANG LIAN \\ State Key Laboratory of Marine Environmental Science, and Department of Physical Oceanography, \\ College of Ocean and Earth Sciences, Xiamen University, Xiamen, China \\ WILLIAM D. SMYTH \\ College of Earth, Ocean and Atmospheric Science, Oregon State University, Corvallis, Oregon \\ ZHIYU LIU
}

State Key Laboratory of Marine Environmental Science, and Department of Physical Oceanography, College of Ocean and Earth Sciences, Xiamen University, Xiamen, China

(Manuscript received 9 September 2019, in final form 23 February 2020)

\begin{abstract}
We explore numerical methods for the stability analysis of stratified, parallel shear flows considering the effects of small-scale turbulence represented by eddy viscosity and diffusivity. The result is an extension of the classical Taylor-Goldstein problem applicable to oceanic and atmospheric flows. Solutions with imaginary frequency describe shear and convective instabilities, whereas those with real frequency represent internal gravity waves. Application to large observational datasets can involve considerable computation and therefore requires a compromise between speed and accuracy. We compare several numerical methods to identify optimal approaches to various problems.
\end{abstract}

\section{Introduction}

The Taylor-Goldstein (T-G) equation describes the stability of a Boussinesq stratified shear flow under inviscid and nondiffusive conditions (Taylor 1931; Goldstein 1931; Liu 2010; Smyth and Carpenter 2019). It is a second-order ordinary differential equation whose solutions exhibit singularity at critical levels (where the speed of the mean current matches the phase velocity). The singular behavior arises from the neglect of viscosity, diffusion and nonlinearity in the equations of motion (Maslowe 1986), and makes the T-G equation a challenge to solve numerically. Several wellknown theorems, such as the Rayleigh inflection point criterion (Rayleigh 1880), Fjørtoft's condition (Fjørtoft 1950), the Miles-Howard theorem (Miles 1961; Howard 1961), and Howard's semicircle theorem (Howard 1961), allow us to predict certain aspects of instability

Corresponding author: Zhiyu Liu, zyliu@xmu.edu.cn without solving the T-G equation, but the information they give is far from complete. One alternative is to use a numerical shooting method together with asymptotic matching to cross singularities (Hazel 1972; Smyth and Peltier 1989; Rees and Monahan 2014). Another is to retain the viscous and diffusive effects. Liu et al. (2012) extended the T-G equation to include vertical and horizontal eddy coefficients of viscosity and diffusivity, allowing those coefficients to vary with height. The resulting viscous T-G (vT-G) equation set (described below) is useful for modeling naturally occurring flows. It is nonsingular, but it is higher order than the T-G equation.

The focus of this paper is numerical methods for the vT-G equations, specifically matrix-based methods. Matrix methods are slower than shooting methods for a given accuracy but are useful in practice because they do not rely on an initial guess. We will compare several commonly used methods to seek the most efficient way to get a solution with a prescribed level of accuracy. 


\section{The viscous Taylor-Goldstein problem}

For a small-amplitude, normal mode disturbance with horizontal wave vector $\mathbf{k}$ evolving on a horizontal background flow $\mathbf{U}_{h}=\{u(z), v(z)\}$, mode evolution is determined solely by the component of the background flow parallel to the wave vector:

$$
U=\frac{\mathbf{k} \cdot \mathbf{U}_{h}}{k},
$$

where $k=\sqrt{\mathbf{k} \cdot \mathbf{k}}$ is the wave vector magnitude. Therefore, without loss of generality, we consider the two-dimensional problem that the parallel shear flow $U(z)$ is disturbed by the vertical velocity perturbation $w^{\prime}$ and the buoyancy perturbation $b^{\prime}$. The buoyancy is defined as $b=$ $g\left(\rho_{0}-\rho\right) / \rho_{0}$, where $g$ is the acceleration due to gravity, and $\rho_{0}$ is a characteristic value of the density $\rho$. We decompose the density as $\rho=\rho_{0}+\bar{\rho}(z)+\rho^{\prime}(x, y, z, t)$, and the buoyancy becomes $b=B(z)+b^{\prime}$, where $B=-g \bar{\rho} / \rho_{0}$ and $b^{\prime}=-g \rho^{\prime} / \rho_{0}$ (see appendix E). The perturbations can be expressed as the normal mode

$$
w^{\prime}=\left[\hat{w}(z) e^{\sigma t+\imath k x}\right]_{r} ; \quad b^{\prime}=\left[\hat{b}(z) e^{\sigma t+\imath k x}\right]_{r},
$$

where $\hat{w}$ and $\hat{b}$ are complex vertical structure functions of vertical velocity and buoyancy, respectively, $\sigma=\sigma_{r}+\iota \sigma_{\iota}$ is the complex growth rate, subscripts $r$ and $i$ indicate the real and imaginary parts, $\iota=\sqrt{-1}$ and $x$ is the horizontal coordinate parallel to the wave vector. We include the effects of viscosity and diffusivity, which may either be molecular in origin or result from turbulence on spatial scales much smaller than the wave scale $k^{-1}$. Following Liu et al. (2012), the viscosity and diffusivity have separate horizontal $\left(A_{h}, K_{h}\right)$ and vertical $\left(A_{v}, K_{v}\right)$ parts, and are allowed to vary in the vertical. The linearized normal-mode equations are then

$$
\begin{aligned}
(\sigma+\iota k U) \nabla^{2} \hat{w}-\iota k \frac{d^{2} U}{d z^{2}} \hat{w} & =T_{w} \hat{w}-k^{2} \hat{b}, \\
(\sigma+\iota k U) \hat{b}+\frac{d B}{d z} \hat{w} & =T_{b} \hat{b},
\end{aligned}
$$

with viscous and diffusive operators

$$
\begin{aligned}
& T_{w}=\frac{d^{2}}{d z^{2}}\left(A_{v} \frac{d^{2}}{d z^{2}}\right)-k^{2} \frac{d}{d z}\left[\left(A_{h}+A_{v}\right) \frac{d}{d z}\right]+k^{4} A_{h}, \\
& T_{b}=\frac{d}{d z}\left(K_{v} \frac{d}{d z}\right)-k^{2} K_{h} .
\end{aligned}
$$

The Boussinesq approximation has been made to neglect the effect of variation of density except in the buoyancy. In the inviscid, nondiffusive limit $A_{h}=K_{h}=A_{v}=K_{v}=0$, the vT-G equations [Eqs. (1) and (2)] become the conventional T-G equation.

The vT-G equations [Eqs. (1)-(4)] can be restructured as a generalized differential eigenvalue problem:

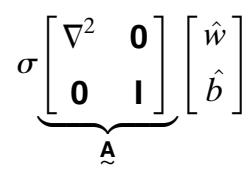

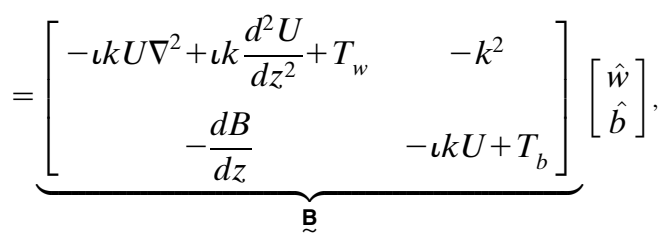

where $\mathbf{0}$ is the zero matrix, $\mathbf{I}$ is the identity matrix, and

$$
\nabla^{2}=d^{2} / d z^{2}-k^{2}
$$

is the Laplacian in normal-mode form and $\underset{\sim}{\mathbf{A}}$ and $\underset{\sim}{\mathbf{B}}$ are matrix differential operators.

Specification of the problem is completed by the imposition of boundary conditions at the upper and lower boundaries, which may in principle be at infinity. Each boundary is assumed to be impermeable $(\hat{w}=0)$, either rigid $(d \hat{w} / d z=0)$ or frictionless $\left(d^{2} \hat{w} / d z^{2}=0\right)$ and either with constant buoyancy $(\hat{b}=0)$ or insulating $(d \hat{b} / d z=0)$.

\section{Numerical methods}

The problem specified by (5) and the boundary conditions is to find (complex) eigenvalues $\sigma$ for given profiles of $U(z), B(z), A_{v}(z), A_{h}(z), K_{v}(z)$, $K_{h}(z)$, and wavenumber $k$. We begin by defining grid points $z_{i}(i=1, \ldots, N)$, which may or may not be evenly spaced (appendix A). We then define derivative matrices $\underline{\mathbf{D}}^{(K)}$ such that the $K$ th derivative of a discretized function $f_{i}=f\left(z_{i}\right)$ is represented by

$$
\left.\frac{d^{K} f}{d z^{K}}\right|_{z=z_{i}}=D_{i j}^{K} f_{j}
$$

The differential eigenvalue problem (5) now becomes the generalized eigenvalue problem

$$
\sigma \underset{\sim}{\mathbf{A}} \mathbf{x}=\underset{\sim}{\mathbf{B}} \mathbf{x}
$$

where $\underset{\sim}{\mathbf{A}}$ and $\mathbf{B}$ are $2 N \times 2 N$ matrices and the eigenvector $\tilde{\mathbf{x}}$ is the concatenation of the elements of the discretized structure functions $\hat{w}_{i}$ and $\hat{b}_{i}$. This algebraic problem is solvable using standard numerical routines, 
but we need first to specify the derivative matrices ${\underset{\sim}{(K)}}^{(K)}$ that appear in $\underset{\sim}{\mathbf{A}}$ and $\underset{\sim}{\mathbf{B}}$.

Numerical methods for determining ${\underset{\mathbf{D}}{(K)}}^{(K)}$ can be classified into the local and global categories. For the local methods, the derivative at the grid point $z_{i}$ involves only a few neighboring function values $f\left(z_{j}\right)$. We can then calculate $D_{i j}^{(K)}$ by applying Taylor's expansion of $f$ or by differentiating a local polynomial interpolant at $z_{i}$ (appendix B). The result is a sparse matrix with bandwidth $J$. The larger $J$ is, the higher the order of $\underline{\mathbf{D}}^{(K)}$ is.

In global methods, computing the derivative at any point involves all the $N$ nodes in the domain. Here, $D_{i j}^{(K)}$ can be derived by differentiating a high-order orthogonal polynomial such as the Chebyshev polynomial (appendix C) or integrating a trigonometric series (appendix D). As a result, $\mathbf{D}^{(K)}$ is a full matrix. This increases the CPU time and storage requirements but, in compensation, the orthogonal high-order polynomials give higher accuracy for a given $N$. Details of the computation of the differentiation matrices are given in the appendixes.

It is important to recognize that higher-order finitedifference methods are designed to be more accurate in the limit $\Delta \rightarrow 0$, where $\Delta$ is the grid spacing defined in appendix A. In practice, we make $\Delta$ as small as we can with the available resources, but often we are interested in marginally resolved flow features, for example, a layer of strong shear that extends over just a few data points. In this case, there is no guarantee that a higher-order method will be more accurate. This failure of higher-order methods to improve accuracy has been noted previously, for example, by Putrevu and Svendsen (1992).

In some applications, our aim is to approximate the solution of the differential eigenvalue problem as accurately as possible, while in others we try to attain acceptable accuracy while minimizing processing time and memory. Therefore, we will compare methods based on two criteria:

- How quickly does the error of the growth rate (or phase speed) go to zero as $N \rightarrow \infty$ ?

- What is the smallest $N$ needed to achieve a predetermined accuracy, for example, a relative error of $1 \%$ in the growth rate?

We will address these questions for a set of test problems including both simple, idealized models of parallel flow and in situ oceanographic measurements.

\section{Shear instabilities in idealized model flows}

Our first goal in solving the vT-G equations is to identify unstable normal modes, or instabilities. We will begin with a profile that is often used in

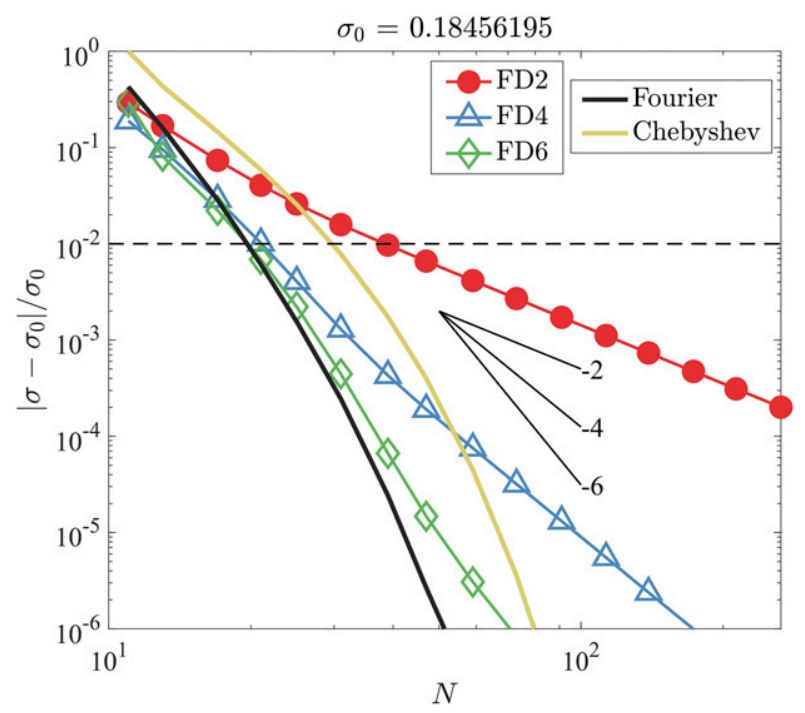

FIG. 1. Relative error in the growth rate $\sigma$ for case 1 with different numerical methods as shown in the legend.

modeling geophysical flows, the hyperbolic tangent shear layer.

\section{a. Case 1: A homogeneous, inviscid shear layer}

In the homogeneous, inviscid limit the vT-G equation reduces to the Rayleigh equation (Rayleigh 1880; Smyth and Carpenter 2019). We consider a hyperbolic tangent shear profile $U=\tanh (z)$ with boundary conditions $\hat{w}=0$ at $z= \pm H / 2$, where $H$ is the domain height, chosen to be 10 . Further boundary conditions are not needed since $T_{w}$ and $T_{b}$ drop out of Eqs. (5). For each wavenumber $k$, a matrix-based method (local or global; see appendixes B-D) using $N$ grid points delivers $2 N$ eigenmodes. We are concerned only with the fastest growing of these eigenmodes, that is, $\sigma_{\mathrm{FGM}}$.

The growth rate is purely real, and is a maximum when $k=0.46$. As a ground truth for testing the various numerical methods, we use the Fourier-Galerkin method (appendix C) with high resolution $N=801$ to find the corresponding growth rate $\sigma_{0}=0.18456195$. This is used as a ground truth, or "target value" to define the relative error, $\left|\sigma-\sigma_{0}\right| / \sigma_{0}$, for each of the other methods tested.

Figure 1 shows the relative error in the growth rate for five methods: the second-, fourth-, and sixth-order finitedifference methods (labeled FD2, FD4 and FD6, respectively) and the Fourier and Chebyshev methods. The number of grid points $N$ ranges from 11 to 265 . Not surprisingly, all methods become more accurate as $N$ is increased. The error in the finite-difference methods decreases according to the order of the method, giving very accurate results at high $N$. At low $N$, however, the difference vanishes. To achieve $1 \%$ accuracy in the 
growth rate requires $N=39,21$, and 19 with FD2, FD4, and FD6, respectively. The global methods become extremely accurate at large $N$, but do not perform as well at small $N$ (the Chebyshev method especially). The standard of $1 \%$ accuracy requires $N=29$ and 19 with the Chebyshev and Fourier methods, respectively. An investigator desiring $<1 \%$ error in the growth rate with high efficiency might do well to choose FD6 or the Fourier method. However, FD4 is simpler while giving nearly the same accuracy.

We now show that clustering the grid points in regions of high shear using the methods described in appendix A can improve accuracy significantly, especially when the resolution is coarse (Fig. 2). In general, the clustered grid delivers greatly improved accuracy for all orders of finite-difference methods. The difference is particularly striking at low $N$, where the $1 \%$ standard is achieved even for the lowest-order method FD2c, where "c" denotes clustering (not to be confused with the phase speed of internal waves in section 6) and at the lowest resolution tested $(N=11)$.

For each of the computations shown in Fig. 2 that used clustered grids, an optimal value was chosen for the clustering parameter $a$ (see appendix A). This was done by maximizing an improvement index $\mathrm{Y}$, which compares the accuracy achieved with a particular clustered grid with that using a uniform grid:

$$
\Upsilon(a, N)=1-\left|\frac{\sigma_{\mathrm{FDc}}-\sigma_{0}}{\sigma_{\mathrm{FD}}-\sigma_{0}}\right|,
$$

where $\sigma_{\mathrm{FD}}$ is the growth rate computed using a particular finite-difference method with uniform grids and $\sigma_{\mathrm{FDc}}$ is the value obtained using clustered grids.

To illustrate the method, we examine the dependence of $\Upsilon$ on $a$ at $N=113$ (Fig. 3a). Using method FD2c, accuracy improves steadily as $a$ is increased, reaching a maximum at $a=0.85$. We identify this as the optimal value for FD2c. With method FD4c, the increase is faster and the maximum improvement is found at $a=$ 0.60 . With method FD6c, maximum improvement is also found at $a=0.60$, but for higher values of $a$, the rate of improvement decreases approaching zero as the clustering becomes extreme.

We next examine the optimal value of $a$ for a range of $N$ (Fig. 3b) using second-, fourth-, and sixth-order methods. It is found that the optimal value of $a$ for FD2c is constant; for FD4c and FD6c, the optimal value of $a$ increases when $N$ is small. These results are important because the advantage of the clustered grid method is greatest for coarsely resolved features.

While the above results might suggest using larger values of $a$, one should recall that setting $a$ too large can

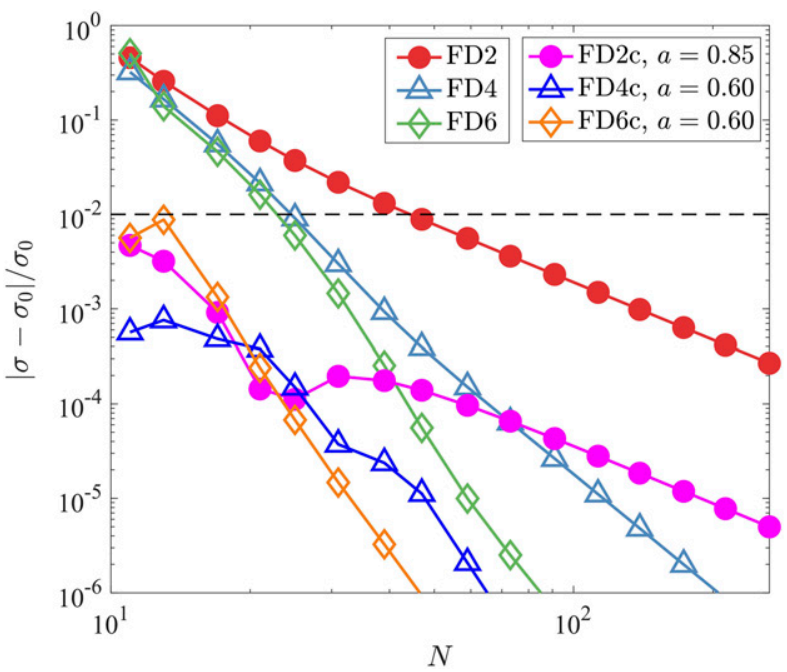

FIG. 2. Relative error in the growth rate for case 1 using finitedifference methods of different order on uniform and nonuniform grids.

degrade accuracy (Fig. 3a). Conservatism is especially well justified when analyzing observational data, where one may not know a priori the flow structures that the code will encounter. On that basis, we tentatively choose the lower values $a=0.85$ for FD2c, and $a=0.60$ for FD4c and FD6c, as in Fig. 2.

We can go one step further and use eigenfunction to test the accuracy of different numerical methods. As we do to the growth rate, the relative error of vertical velocity eigenfunction can be defined as

$$
\Psi(N)=\overline{\left|\frac{|\hat{w}|-|\hat{w}|_{0}}{|\hat{w}|_{0}}\right|},
$$

where $|\hat{w}|$ is the magnitude of the vertical velocity eigenfunction, $|\hat{w}|_{0}$ is the target eigenfunction corresponding to $\sigma_{0}$, and the overline indicates vertical average. As shown in Figs. 4b and 4c, we normalize the solutions by scaling the maximum magnitude of the vertical velocity eigenfunction to unity. The eigenfunction is symmetric about $z=0$, having peaks of equal magnitude on the upper and lower edges of the shear layer. Figure $4 \mathrm{~d}$ shows the relative error $\Psi$ for the FD methods, the FDc methods, and the Fourier and Chebyshev methods. The FDc methods achieve higher accuracy than the FD methods and global methods, indicating that the clustered grids work for eigenfunction. The Chebyshev method performs better than the Fourier method. This is because the collocation points of the Chebyshev method is symmetric about $z=0$, well suitable for this case. We also note that the clustering strength needed to resolve the eigenfunction is smaller 

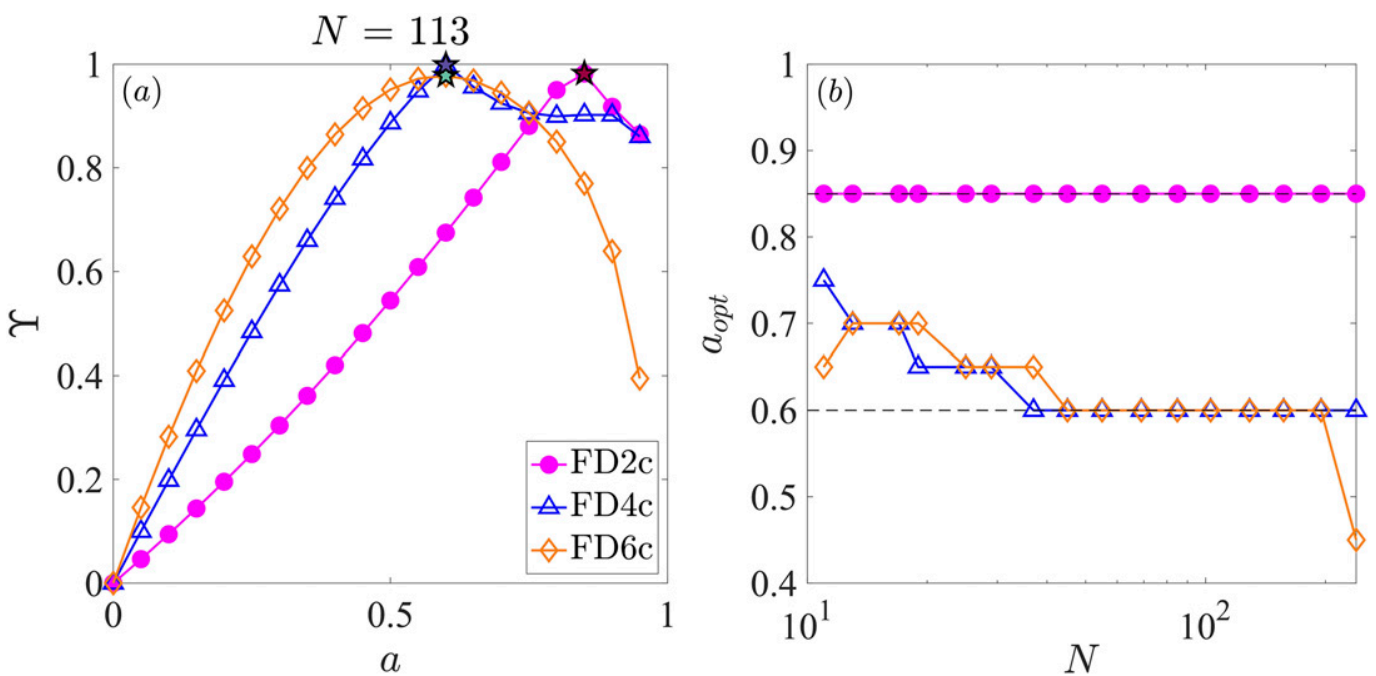

FIG. 3. (a) Improvement index $\Upsilon$ [defined in Eq. (8)] vs the clustering parameter $a$ at $N=113$. The black pentagrams highlight the optimal values. (b) Optimal value of $a$ vs $N$. The dashed lines indicate the convergence value for FD2c, FD4c, and FD6c, which are $0.85,0.60$, and 0.60 , respectively.

than that needed to resolve the growth rate. At $N=11$, FD4c method with $a=0.40$ can reproduce the eigenfunction (dashed line in Fig. 4c).

\section{b. Case 2: A stably stratified, inviscid, nondiffusive shear layer}

We turn now to an inviscid, nondiffusive, stably stratified fluid for which the parallel shear flow problem reduces to the Taylor-Goldstein equation (Taylor 1931; Goldstein 1931; Smyth and Carpenter 2019).
Different numerical methods were tested with a simple case of a shear layer in homogeneous fluid in the previous section. Earth's oceans and atmosphere, however, tend to be density stratified. It is thus critical to make sure these methods also work well for stratified shear flows.

As an example, we consider the hyperbolic tangent model for the stably stratified shear layer:

$$
U(z)=\tanh (z) ; \quad B(z)=\mathrm{Ri}_{b} \tanh (z),
$$
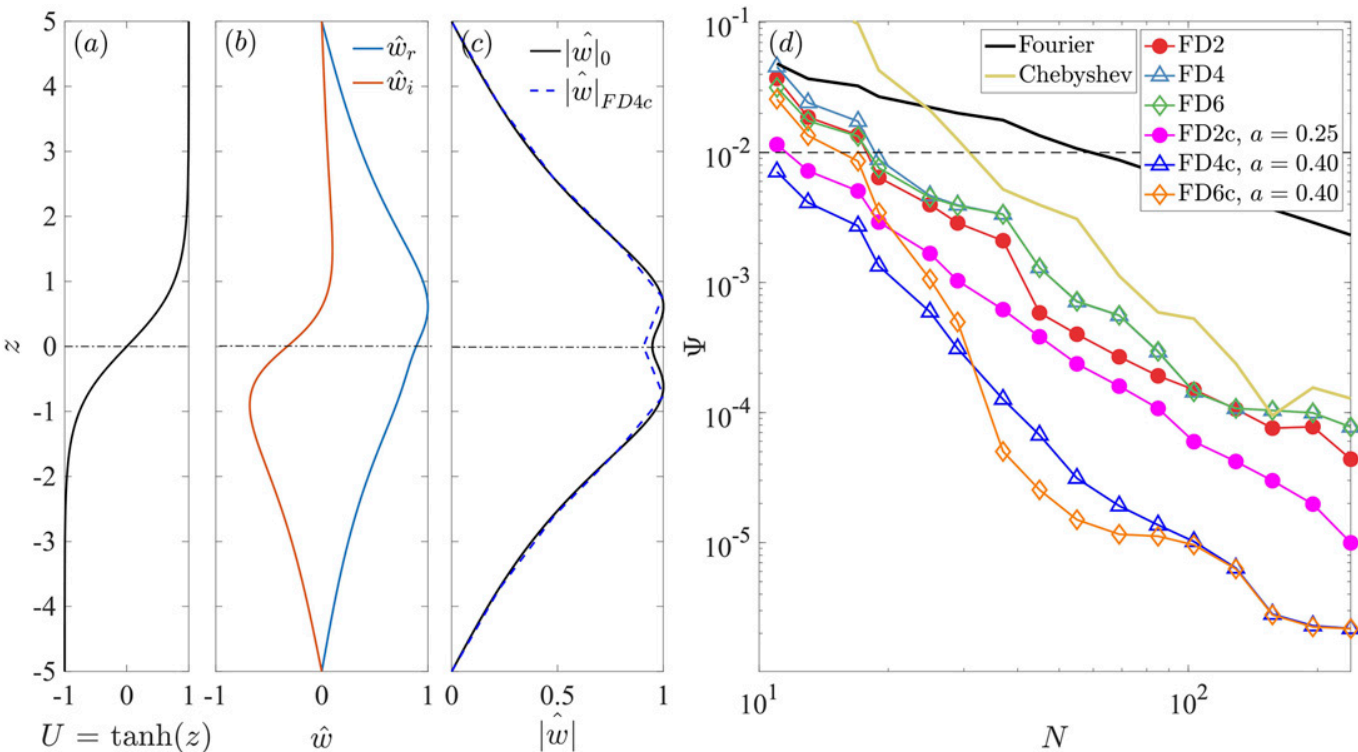

FIG. 4. Eigenfunction of the fastest-growing mode for case 1. (a) Background velocity profile for reference. (b) Real and imaginary parts of eigenfunction $\hat{w}$. (c) Eigenfunction magnitudes for target and the FD4c method with $N=11$. (d) Relative error in the eigenfunction with different numerical methods as shown in the legend. 


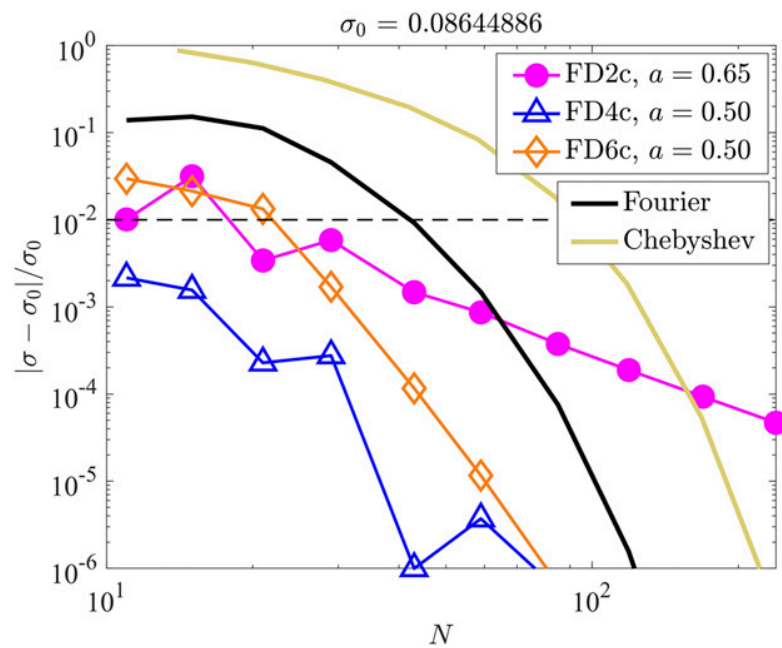

FIG. 5. Relative error in the growth rate $\sigma$ for case 2 with the FDc, Fourier, and Chebyshev methods. As in Fig. 2, but with $\mathrm{Ri}_{b}=0.15$.

where the constant $\mathrm{Ri}_{b}$ is the bulk Richardson number characterizing the strength of the stratification relative to the shear. Differentiating $B(z)$, we have the squared buoyancy frequency

$$
B_{z}(z)=\mathrm{Ri}_{b} \operatorname{sech}^{2}(z) .
$$

In this case, we set $\mathrm{Ri}_{b}=0.15$ and assume that $\hat{w}=0$ and $\hat{b}=0$ at $z= \pm H / 2$ and $H=10$. The target growth rate $\sigma_{0}$ is smaller than that in case 1 (Fig. 5), meaning that shear flow is stabilized by stratification.

We now repeat the calculations for which results were shown in Figs. 1-3. In this case the FDc methods are always more accurate than the FD methods. We therefore neglect the latter and compare the different order FDc methods with the Fourier and Chebyshev methods in Fig. 5. Except at high $N$, FDc methods achieve higher accuracy than the Fourier and Chebyshev methods, with the FD4c method performing best. The optimal values of $a$ are similar to those found in the unstratified case, though not exactly the same (Fig. 5, legend). Once again, high-order FDc methods work best with smaller $a$.

\section{c. Case 3: A homogeneous, stratified shear layer with variable eddy viscosity and diffusivity}

Suppose that the shear layer has experienced instability events in the past and therefore has some residual turbulence represented by the eddy coefficients $A_{v}, A_{h}$, $K_{v}$, and $K_{h}$. We model this scenario as

$$
\begin{aligned}
U(z) & =\tanh (z), \quad B=0 \\
A_{v}(z) & =A_{h}(z)=K_{v}(z)=K_{h}(z)=\operatorname{sech}^{2}(z) / \mathrm{Re},
\end{aligned}
$$

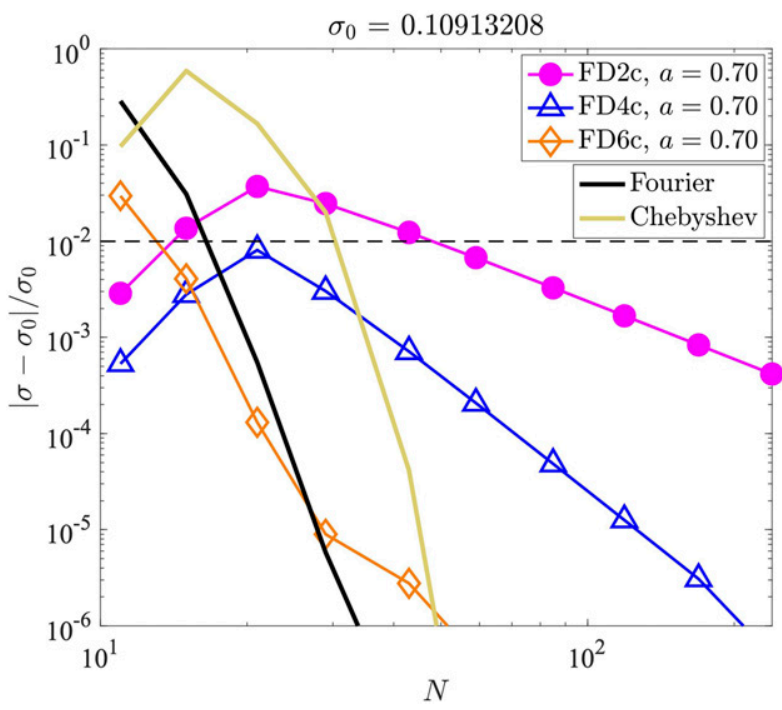

FIG. 6. Relative error in the growth rate $\sigma$ for case 3 using the FDc, Fourier, and Chebyshev methods. As in Fig. 2, but with $\mathrm{Re}=10$

where the constant $\mathrm{Re}$ is the Reynolds number. The eddy coefficients are assumed to be independent of horizontal direction. The turbulent Prandtl number is unity $\left(K_{v}=A_{v}, K_{h}=A_{h}\right)$. We set $\mathrm{Re}=10$ and do the same analyses as in previous cases. Boundary conditions are again $\hat{w}=\hat{w}_{z z}=\hat{b}=0$ at $z= \pm H / 2$ with $H=$ 10 . Use of the normal-mode formalism in viscous, diffusive fluid with arbitrary profiles $U(z)$ and $B(z)$ implies the assumption that the instability grows on a fast time scale in contrast with the slow diffusion of the mean profiles, the so-called frozen flow approximation. This generally requires that $\mathrm{Re}$ be sufficiently large (Smyth and Carpenter 2019). Here, however, we work with smaller Re to provide a rigorous test of the numerical approximations used.

Figure 6 shows the results. Again, the target growth rate is smaller than that in case 1 . This reveals another stabilizing mechanism: shear instability damped by eddy viscosity and diffusion. The clustered finitedifference methods give better accuracy than the global methods at low $N$. At high $N$, the global methods converge rapidly, with the Fourier method outperforming the Chebyshev.

\section{d. Case 4: Plane Poiseuille flow in homogeneous, viscous fluid}

Previous cases have employed frictionless boundaries, with $d^{2} \hat{w} / d z^{2}=0$. We turn now to plane Poiseuille flow, which consists of a parabolic velocity profile between two stationary plates

$$
U(z)=4 z(1-z) .
$$




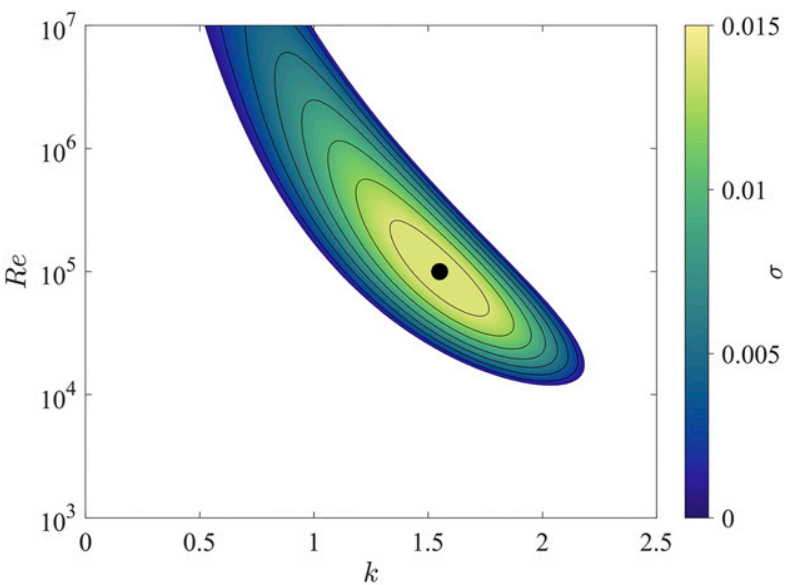

FIG. 7. Growth rate vs wavenumber and Reynolds number for case 4: plane Poiseuille flow with uniform viscosity.

The fluid is assumed to be homogeneous $(B=0)$ and $A_{v}=A_{h}=1 / \mathrm{Re}$. We impose rigid boundary conditions: $\hat{w}=d \hat{w} / d z=0$ at $z=0$ and $z=1$.

The Chebyshev method with resolution $N=101$ is used to compute the growth rate as a function of $k$ and $\operatorname{Re}$ (Fig. 7). For $\operatorname{Re}>10^{5}$, the maximum growth rate increases with decreasing $\mathrm{Re}$, that is, with increasing viscosity. This is the Tollmien-Schlichting instability (Schlichting and Gersten 2016), an example of a mode that is destabilized by viscosity. The growth rate maximum is found at $\mathrm{Re}=10^{5}$ and $k=$ 1.55. Figure 8 shows relative errors of the growth rate at the Reynolds number $\mathrm{Re}=10^{5}$. In this case, both the FDc and Chebyshev methods cluster the grid points in the boundary layers. When resolution is coarse, the strongly clustered FD methods $(a \geq 0.9)$ achieve higher accuracy than the Chebyshev method. The FD6c method performs better than the others. At large $N$, the Chebyshev method converges rapidly. (The Fourier method is omitted because it cannot represent the rigid boundary conditions in the form used here).

To summarize, the numerical methods tested in this paper (see appendixes) compute the growth rate of idealized models of shear instability with varying degrees of accuracy. The accuracy of the finite-difference methods depends on the order of the approximation. When resolution is coarse, clustering the grid in regions of strong shear can confer even higher accuracy than the global Fourier and Chebyshev methods. Most often, higher-order FD methods work best with less clustering (i.e., lower $a$ ). At high $N$, the Fourier and Chebyshev methods converge most rapidly. Before we make a final assessment of all these methods, tests will be carried out with an observed geophysical flows.

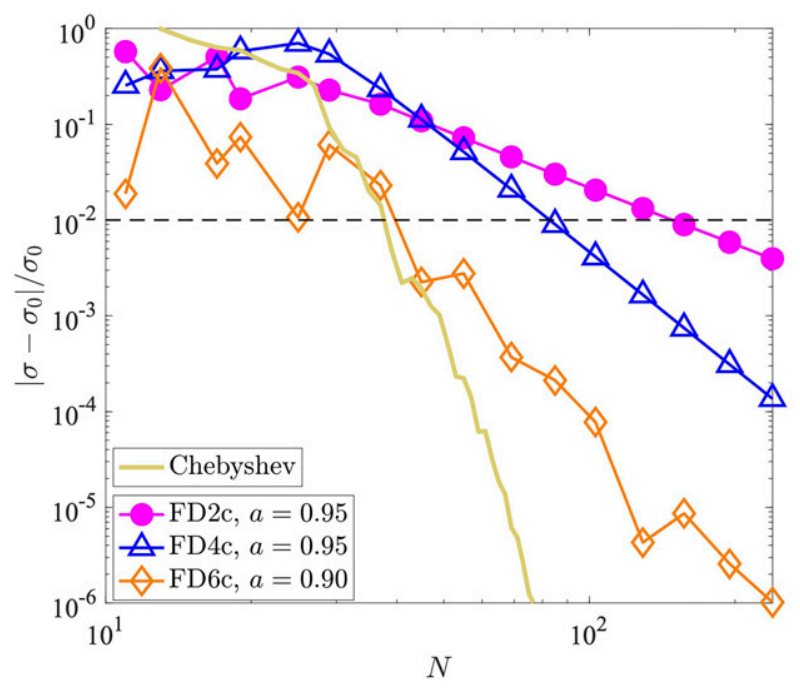

FIG. 8. Relative errors of the growth rate for case 4 with $\mathrm{Re}=10^{5}$ and $k=1.55$ (black dot in Fig. 7).

\section{Shear instabilities in observed oceanic flows}

We now demonstrate the application of these methods to observed geophysical flows. Observed flow profiles are considerably more complicated than the idealized models discussed above (e.g., Sun et al. 1998; Liu 2010; Smyth et al. 2011; Liu et al. 2012; Smyth et al. 2013). In what follows, we consider two cases of increasing complexity.

\section{a. Case 5: Clyde Sea, observed profiles with single shear maximum}

Figure 9 shows the profiles observed at a mooring station $\left(55^{\circ} 21^{\prime} \mathrm{N}, 5^{\circ} 4^{\prime} \mathrm{W}\right)$ in the Clyde Sea at $2000 \mathrm{LT}$ 29 June 2002 (see Liu 2010). The zonal and meridional velocity components were obtained from a moored acoustic Doppler current profiler (ADCP). Because data below $3.6 \mathrm{~m}$ (measured from the bottom) are unreliable due to instrumental constraints, we extrapolate the velocity using cubic splines, assuming $U=V=0$ at the bottom. The profiles of the squared buoyancy frequency $N^{2}$ and diffusivity $K_{v}$ were obtained from a free-fall yo-yo (FLY) microstructure profiler. The diffusivity $K_{v}$ was estimated from the turbulent kinetic energy dissipation rate $\varepsilon$ via

$$
K_{v}=\Gamma \frac{\varepsilon}{N^{2}},
$$

where $\varepsilon$ can be directly estimated from FLY's shear probes signal, and $\Gamma$ is the flux coefficient, commonly approximated by the constant 0.2 (Gregg et al. 2018). As in case 3, the turbulent coefficients are assumed to be isotropic in the horizontal direction 

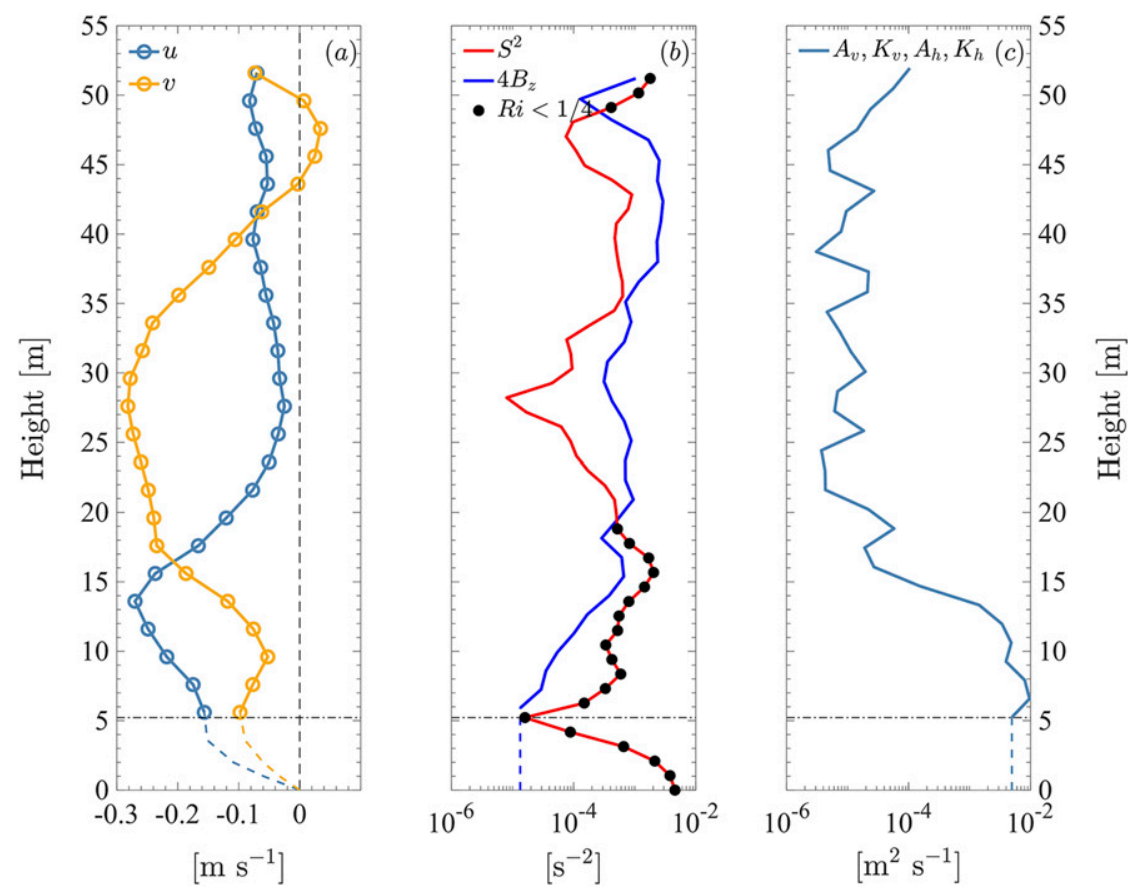

FIG. 9. Background profiles observed at a mooring station $\left(55^{\circ} 21^{\prime} \mathrm{N}, 5^{\circ} 4^{\prime} \mathrm{W}\right)$ in the Clyde Sea at 2000 LT 29 Jun 2002 (case 5). (a) Zonal ( $u$ ) and meridional ( $v$ ) velocity components. Circles indicate the original data and the dashed lines show the extrapolated values. (b) Squared shear $S^{2}=(d u / d z)^{2}+(d v / d z)^{2}$ and fourfold squared buoyancy frequency $4 B_{z}$. The black dots indicate measurement depths where the gradient Richardson number $B_{z} / S^{2}$ is below 0.25 . (c) Turbulent viscosity/diffusivity. Horizontal dot-dashed lines show the lowest extent of the measurements. Values below this depth are extrapolated as described in the text. All panels are plotted vs height above the seabed.

with turbulent Prandtl number unity: $A_{v}(z)=A_{h}(z)=$ $K_{v}(z)=K_{h}(z)$.

Note that the shear profile has five local maxima (excluding boundaries). Only two of these have $\mathrm{Ri}<$ $1 / 4, z=16 \mathrm{~m}$ and $z=8 \mathrm{~m}$. Eddy viscosity is strong at the latter, likely damping shear instability. We therefore anticipate a single dominant mode with critical level near $z=16 \mathrm{~m}$.

To deal with the veering background flow $\mathbf{U}_{h}=\{u(z)$, $v(z)$, we recall that an instability is affected only by the component of the mean flow parallel to its own wave vector $\mathbf{k}$ (section 2). Accordingly, we define the angle $\theta$ as the direction of the wave vector, measured counterclockwise from due west, and loop over $\theta=$ $\left[-90^{\circ}, 90^{\circ}\right]$. For each $\theta$, the velocity profile appearing in the vT-G equations is

$$
U(z, \theta)=u \cos \theta+v \sin \theta .
$$

We also loop over the wavelength $\lambda=2 \pi / k=[1,100] \mathrm{m}$ and ultimately find the direction and wavenumber where the growth rate of the FGM is maximum (Fig. 10, black dot).
To obtain a target value for evaluating different numerical methods, we use the Fourier method with very high resolution, $N=801$, and thus obtain the following values for the FGM: $\theta_{0}=-54^{\circ}, \lambda_{0}=30.880 \mathrm{~m}$ $\left(k_{0}=2 \pi / \lambda_{0}=0.2035 \mathrm{~m}^{-1}\right)$, and $\sigma_{0}=(6.295821+$ $4.879614 \iota) \times 10^{-3} \mathrm{~s}^{-1}$. The critical level is located at $15.8 \mathrm{~m}$, within the single maximum shear layer.

Figure 11 shows the comparison between the FD methods and the global methods (i.e., the Fourier and Chebyshev methods). At low $N$, no difference is visible between the Fourier and FD methods. The Fourier and FD6 method have higher accuracy than the others at large $N$. The Chebyshev method performs worst, even compared with FD2. This is because the Chebyshev method clusters the grid points near the boundaries, which is inefficient for this case.

We cluster the grid points in the single shear layer and compare the accuracy of the FDc methods of different orders with the FD method on a uniform grid. The FDc method achieves higher accuracy than the FD method (Fig. 12), indicating that the clustered grids work for observed flows. At large $N$, FD6c with $a=0.30$ performs the best. 


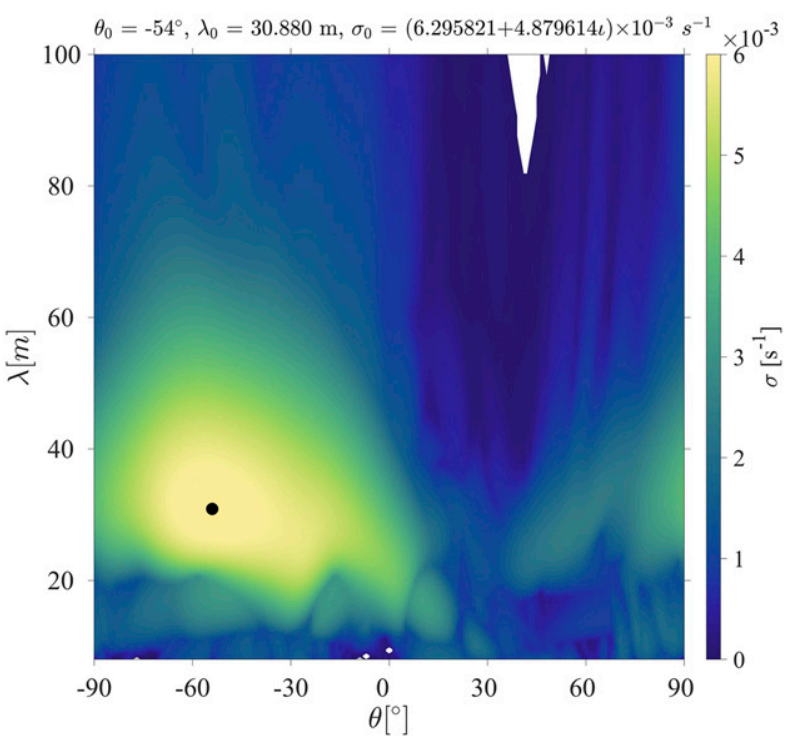

FIG. 10. Growth rate $\sigma$ on the $\theta-\lambda$ space for case 5. Here, $\theta$ is the wave vector direction and $\lambda$ is the wavelength. For each $\mathbf{k}$, growth rate is calculated using the Fourier method with high resolution with $N=801$. The black dot indicates the fastest-growing mode. The title shows the maximum value of $\sigma$ and the corresponding wavenumbers.

\section{b. Case 6: Clyde Sea, observed profiles with multiple shear maxima}

Observed profiles are often more complex than the one shown above, for example, Fig. 13. Conditions for shear instability are evident at several depths; that is,

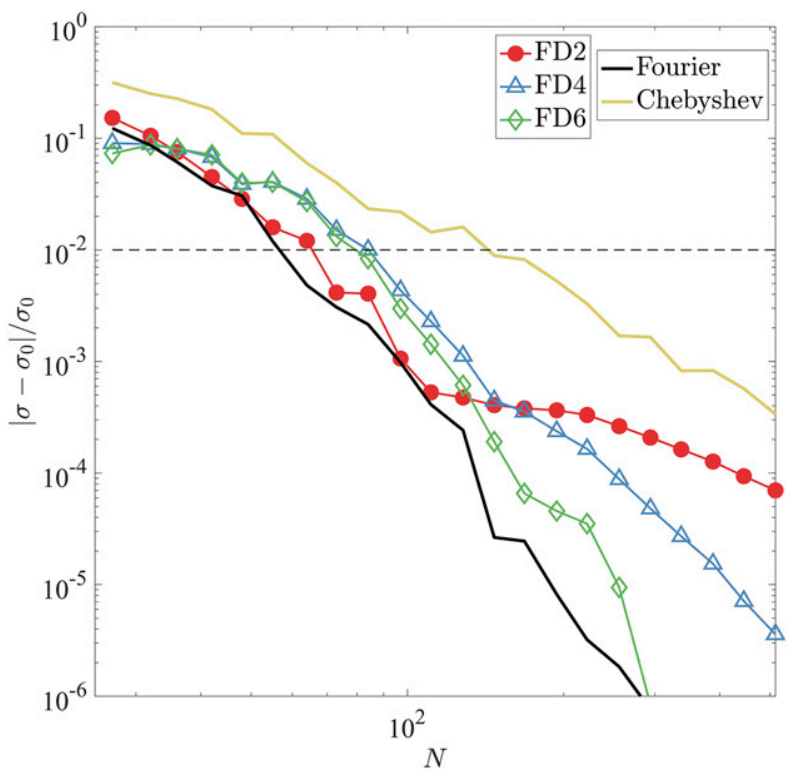

FIG. 11. Relative error in the growth rate $\sigma$ for the FD, Fourier, and Chebyshev methods applied to case 5 .

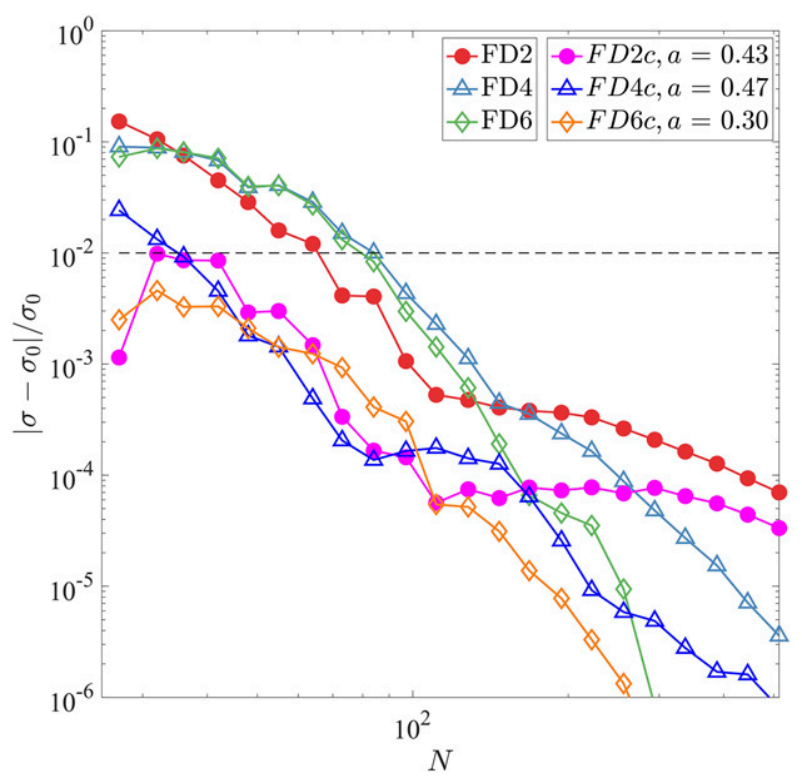

FIG. 12. Relative error in the growth rate $\sigma$ for the FD and FDc methods of different order applied to case 5 . The value of $\sigma$ is computed by Eq. (7) with targeted $\theta$ and $\lambda$ shown in Fig. 10.

shear magnitude reaches a local maximum (red curve in Fig. 13b), $\mathrm{Ri}<1 / 4$ (black dots), and turbulent coefficients are not too large (Fig. 13c). In this case the stability analysis at each $(k, \theta)$ may deliver two or more unstable modes with critical levels close to one of the shear maxima shown in Fig. 13b. We describe a method for dealing with this additional complexity. At each $(k, \theta)$, rather than retain only the fastest growing of the $2 \mathrm{~N}$ eigenmodes, we collect all modes having positive growth rates. After scanning the $k-\theta$ plane, we are typically left with several thousand modes.

Following Smyth et al. (2013), we compute the critical level for each mode and plot a histogram of the results (Fig. 13d). For the case analyzed here, the histogram reveals eight peaks, each of which corresponds roughly to one of the shear maxima shown Fig. 13b. Local minima of the histogram (blue triangles in Fig. 13d) conveniently delineate the set of modes corresponding to each shear maximum. We call these sets mode families. Each mode family has its own fastest-growing mode (e.g., pentagrams in Fig. 14a) and critical level (Fig. 14c). The depth of the critical level for each family is approximately independent of wavelength. Eight depths are found and shown by horizontal lines on Fig. 14c. The shallowest critical level, located at $z=49 \mathrm{~m}$, is denoted as mode 1 . Successive mode families with critical levels at $z=45,31,23,19,12,7$, and $1 \mathrm{~m}$ are denoted as modes $2-8$, respectively. These mode families represent distinct 

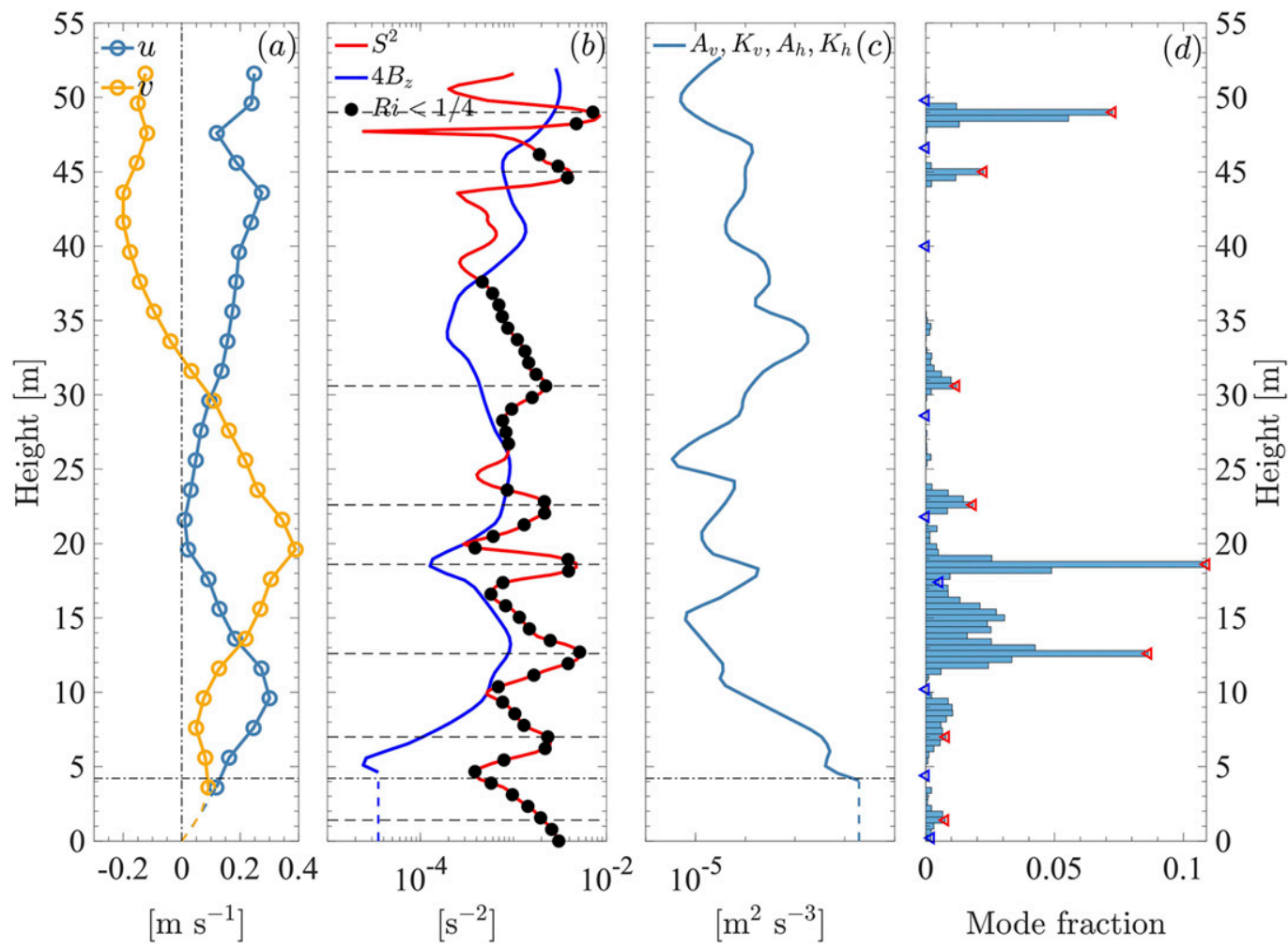

FIG. 13. Case 6: Background profiles observed at a mooring station $\left(55^{\circ} 21^{\prime} \mathrm{N}, 5^{\circ} 4^{\prime} \mathrm{W}\right)$ in the Clyde Sea at 0300 LT 30 Jun 2002. (a) Zonal $(u)$ and meridional $(v)$ velocities. (b) Squared shear $S^{2}$, fourfold squared buoyancy frequency $4 B_{z}$, and the gradient Richardson number, which is below a quarter. (c) Turbulent viscosity and diffusivity. (d) Histogram of the critical level having positive growth rates. The red and blue triangles show all mode families that are found. The horizontal dash-dotted lines in (a)-(c) show the highshear layers induced by extrapolated velocity. The dashed lines in (b) represent the critical-level depth of mode families found in $(\mathrm{d})$.

physical processes (Sun et al. 1998). For example, the uppermost mode family may represent shear at the base of the surface mixed layer, while others might represent breaking depths of the internal wave field. The growth rates of these mode families are shown on Fig. 14a. Three mode families stand out are mode 1 , mode 5 and mode 6. The growth rates of their FGMs are $39.31,39.76$, and $34.21 \mathrm{~h}^{-1}$, respectively, so their amplitudes grow by a factor $e=2.718$ in about $2 \mathrm{~min}$. The corresponding wavelength are $7.78,12.04$, and $25.43 \mathrm{~m}$, about 7 times the thickness of their shear layer, that is, 1 , 2 , and $4 \mathrm{~m}$.

Figure 15 shows the eigenfunctions of the three dominant modes. The solutions were normalized by scaling the maximum magnitude to unity. The vertical structure of eigenfunctions have significant magnitude at their critical-level depth, while the magnitude decreases to zero away from these layers.

We use mode 6 to test the accuracy of different numerical methods in computing the growth rate of fastest-growing mode $\sigma$, the wavelength $\lambda$, the wave vector direction $\theta$, and the critical-level height $z_{c}$. All methods converge to the same values (blue arrows in Fig. 16). At large $N$, the FD4c method performs as well as the Fourier-Galerkin method and even better than the Chebyshev method. At low N, FD4c achieves the highest accuracy.

\section{Internal gravity waves in inviscid, nondiffusive fluid}

Internal gravity waves arise from the same Eqs. (1) and (2) that describe instabilities. The phase speed of internal gravity waves is $c=\iota \sigma / k=c_{r}+\iota c_{\iota}$, where $\sigma$ is the eigenvalue of Eq. (5). Here, we look for the stable wave solutions $\left(c_{\iota}=0\right)$ and test the abilities of various numerical methods to approximate the phase speed $c_{r}$ in the long-wave (or hydrostatic) limit $k \rightarrow 0$.

We consider a viscous stratified fluid with stratification given by (11) as in case 3 but with $\mathrm{Ri}_{b}=1$ and no background flow, $U=0$. Eddy coefficients are 

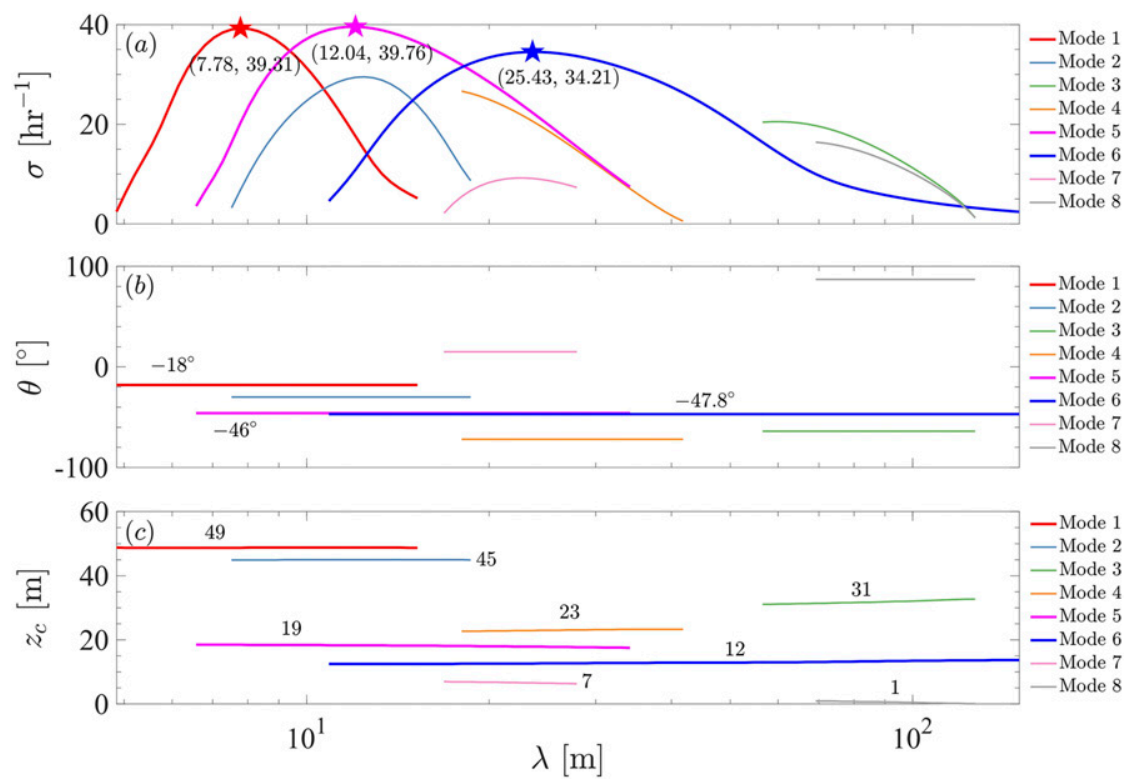

FIG. 14. (a) Growth rates, (b) wave vector direction, and (c) critical-level depths vs wavelength for the eight mode families for case 6 . Pentagrams show the fastest-growing mode of three main mode families.

uniform and equal: $A_{v}=K_{v}=A_{h}=K_{h}=1 /$ Re with $\operatorname{Re}=10^{10}$.

Figure 17 shows the relative error of phase speed. The accuracy of high-order finite-difference methods and global methods is high. Unlike the behavior in Fig. 1, the Fourier method works very well at low $N$. To achieve $1 \%$ accuracy, only 12 grid points are required. The FD4, FD6, and Chebyshev methods have
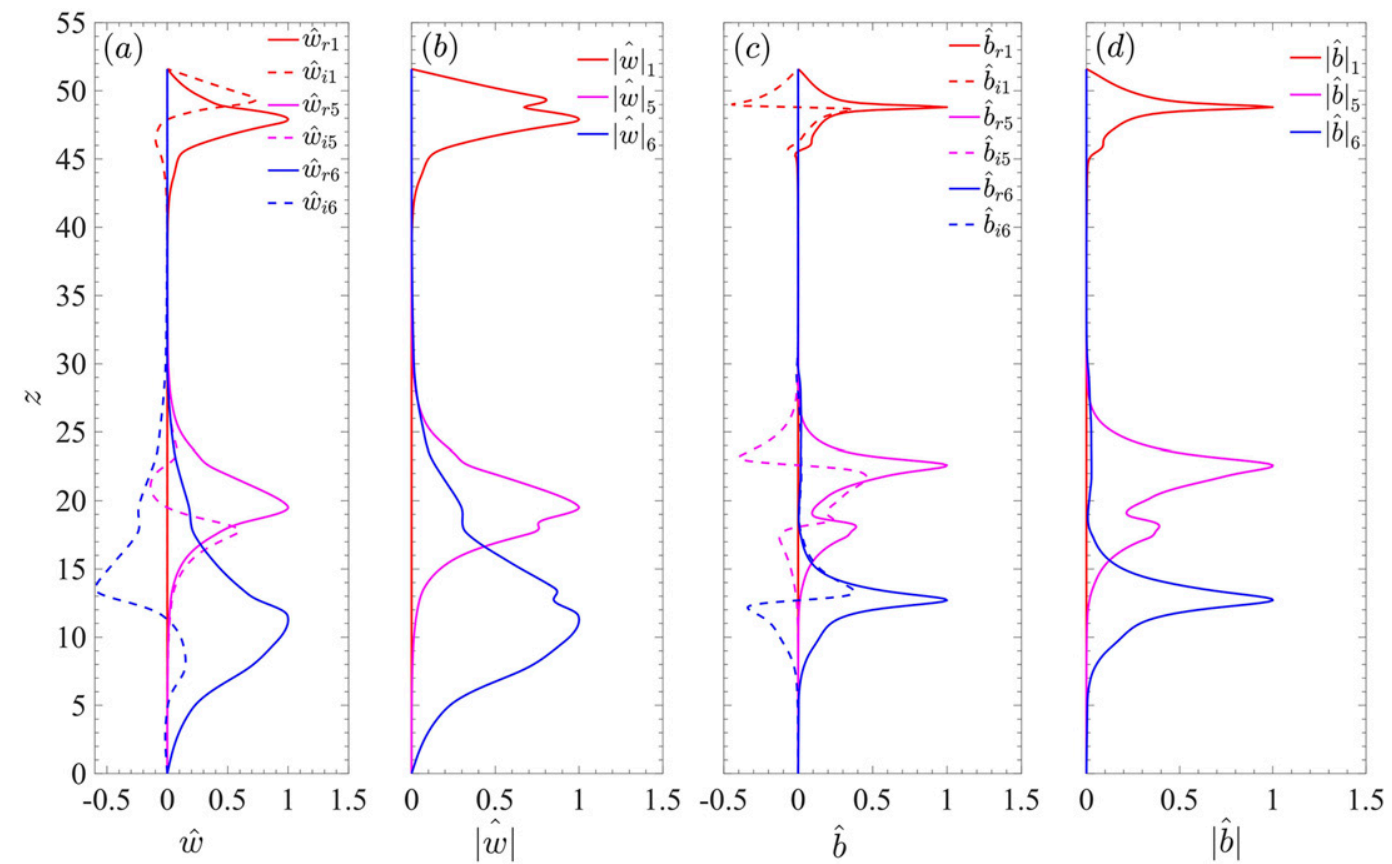

FIG. 15. Eigenfunctions of modes 1, 5, and 6 for case 6. (a) Vertical velocity eigenfunction. (b) Vertical velocity eigenfunction magnitudes. (c) Buoyancy eigenfunction. (d) Buoyancy eigenfunction magnitudes. The maximum magnitude of the vertical velocity and buoyancy of each mode is normalized to unity. 

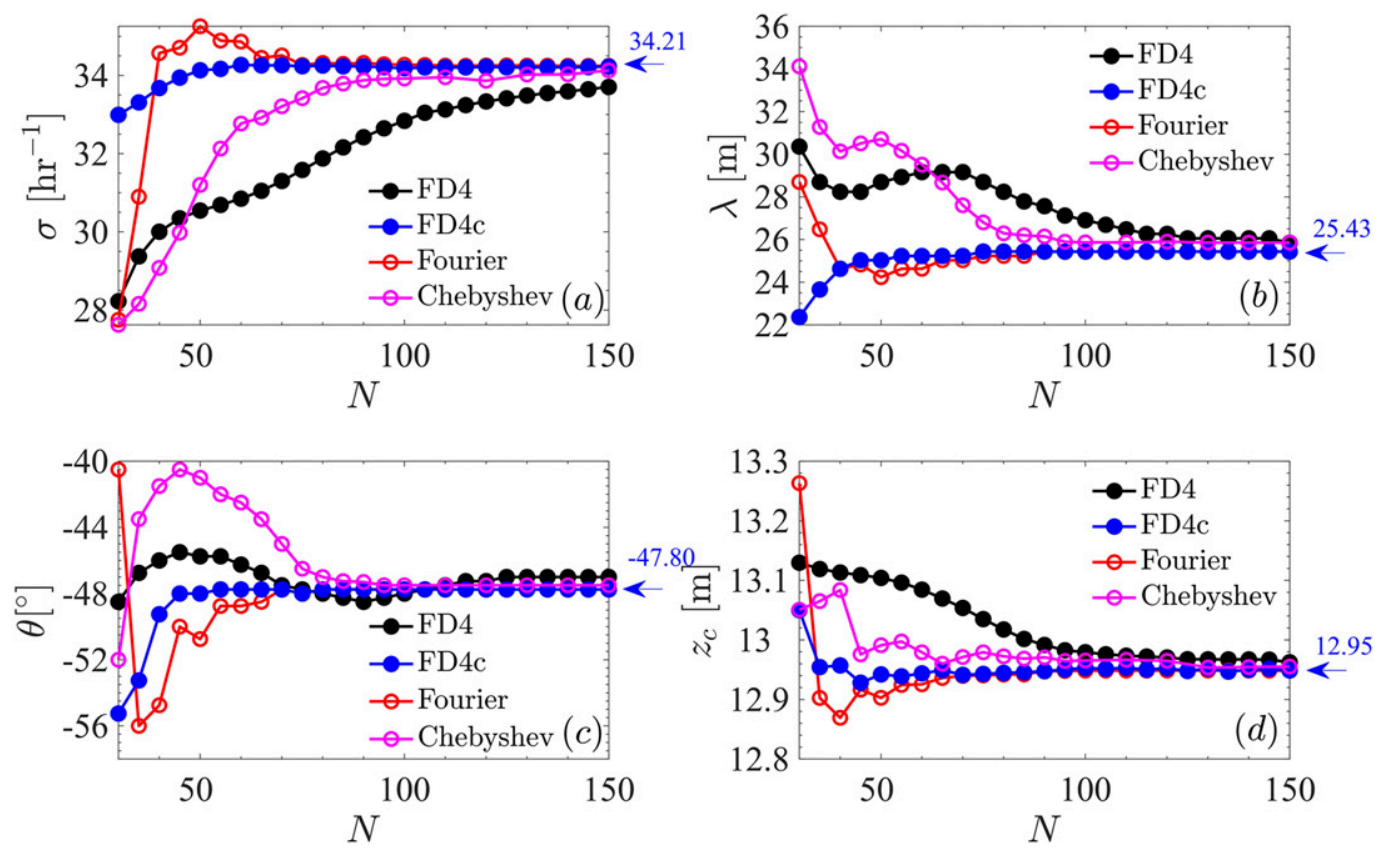

FIG. 16. Numerical methods' ability to catch the characteristic parameter of mode family 6 for case 6. (a) Growth rate of the fastest-growing mode $\sigma$ vs number of grid points $N$. (b) Corresponding wavelength $\lambda$ vs $N$. (c) Corresponding wave vector direction $\theta$ vs $N$. (d) Critical level $z_{c}$ vs $N$. The blue arrows and values on the righthand edge of each plot indicate the convergency values of $\sigma, \lambda, \theta$, and $z_{c}$.

similar accuracy at low $N$. These methods all outperform $\mathrm{FD} 2$, which requires $N>80$ to achieve $1 \%$ accuracy.

The optimal value of $a$ (Fig. 18b) is generally smaller than the values computed for the homogeneous shear layer (case 1; Fig. 2). The phase speed of internal waves does not seem to require highly clustered grid points; in fact, the FD6 method gives better results with no clustering except when the resolution is very coarse (Fig. 18b; compare green and yellow diamonds). We understand this by recalling that, for shear instability, a high value of $a$ ensures high resolution around the critical level, where the instability is most likely to occur. The neutral modes found here are not singular and are therefore less tightly localized than the instabilities discussed previously. The Fourier method (black curve on Fig. 17) provides reliably accurate solutions.

\section{Summary}

The conventional Taylor-Goldstein equation is extended to include effects of turbulence represented by the eddy viscosity and diffusivity. The resulting "viscous T-G" (vT-G) equation, together with appropriate boundary conditions, is a linear eigenvalue problem that can be solved using matrix-based methods. We have tested the most commonly used matrix methods including the second-, fourth-, and sixth-order finite-difference methods, the Chebyshev-collocation method, and the Fourier-Galerkin method. The accuracy of the finitedifference method is greatly improved by using adaptive grid spacing.

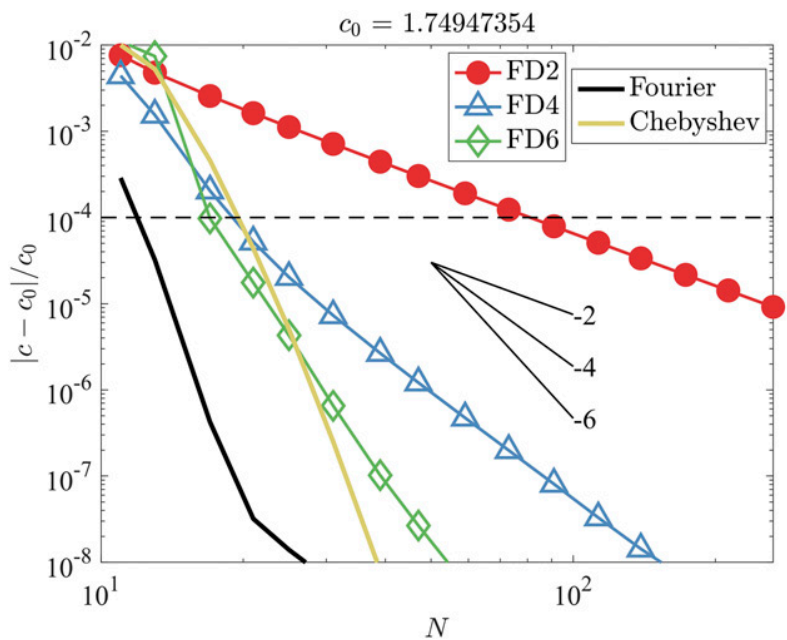

FIG. 17. Relative error in the internal gravity wave speed $c$. The target speed $c_{0}$ is defined using the Fourier-Galerkin method with $N=801$. For other methods with smaller $N, c$ is computed from Eq. (5) with Eq. (11), $k=10^{-6}, U=0, \operatorname{Re}=10^{10}, \operatorname{Pr}=1$, and $\mathrm{Ri}_{b}=1$. 

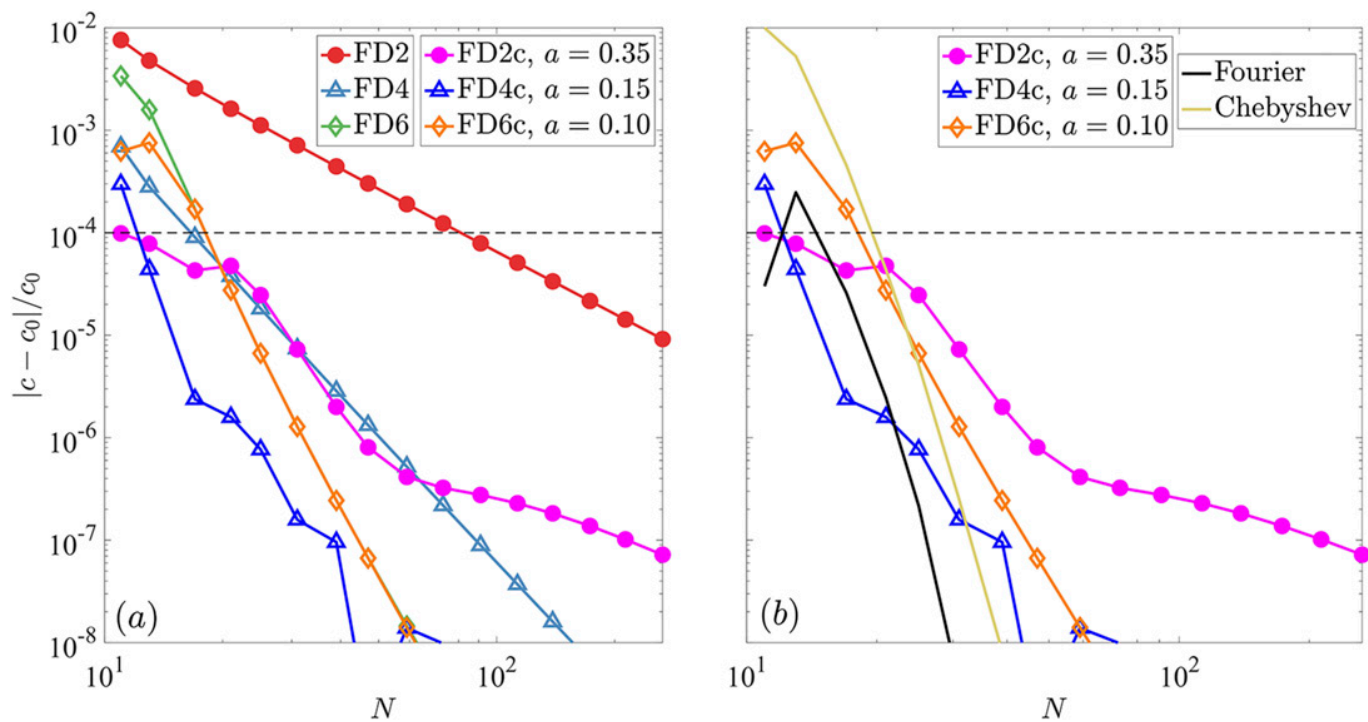

FIG. 18. (a) Relative error in the internal gravity wave speed $c$ for the FD and FDc methods of different order. (b) Relative error in the internal gravity wave speed $c$ for the FDc, Fourier, and Chebyshev methods.

We have tested these methods using both idealized models and observational data. At high resolution, the global methods (i.e., the Fourier-Galerkin and Chebyshevcollocation methods) converge most rapidly. At coarse resolution, however, the finite-difference methods with optimal grid spacing can achieve higher accuracy than the global methods. The optimal value of clustering strength depends on the order of the methods. High-order finite-difference methods usually need only slight clustering. Overall, we recommend the FD $4 c$ method with $a=0.6$ or the FD $6 c$ method with $a=0.4$ for sheared flows. If shear is judged to be unimportant, the FourierGalerkin method is a reliable alternative.

Acknowledgments. This work was supported by the National Natural Science Foundation of China (41622601, 91858201, and 41721005), the National Key R\&D Program of China (2016YFC1401404), and the U.S. National Science Foundation (OCE-1537000). The project has benefited from discussions with Shuwen Tan and Larry Pratt.

Data availability statement: The codes developed in this study are available on request from W.D.S (bill.smyth@ oregonstate.edu) or Z.L. (zyliu@xmu.edu.cn).

\section{APPENDIX A}

\section{Creation of an Efficient Clustered Grid}

Efficient solution of Eqs. (1)-(4) requires an efficient means of discretization; that is, grid spacing can vary with $z$ such that the spacing is fine in regions where the solution is expected to vary most rapidly. Since instability tends to be focused in high-shear layers, it is natural to seek for a discretization with clustering in these regions.

We begin with a set of points spaced evenly between the boundaries $z_{B}$ and $z_{T}$ :

$$
z_{i}=z_{B}+(i-1) \Delta, \quad \text { where } i=1,2, \ldots, N
$$

and

$$
\Delta=\frac{z_{T}-z_{B}}{N-1}
$$

Now suppose, for example, that we want to concentrate resolution in parts of the profile where the shear is strong. We therefore define the absolute shear

$$
s_{i}=\frac{\left|U_{i+1}-U_{i}\right|}{z_{i+1}-z_{i}}, \text { for } \quad i=1,2, \ldots, N-1
$$

and a transformed version

$$
\zeta_{i}=\frac{\ln \left(s_{i} / s_{\min }\right)}{\ln \left(s_{\max } / s_{\min }\right)}
$$

The latter variable approaches 0 and 1 when the absolute shear is at its minimum and maximum values, $s_{\min }$ and $s_{\max }$, respectively. We now define the new grid increment 


$$
\eta_{i}=\frac{z_{T}-z_{B}}{\sum_{k=1}^{N-1}\left(1-a \zeta_{k}\right)}\left(1-a \zeta_{i}\right), \quad i=1,2, \ldots, N-1,
$$

where the stretching parameter $a$ is an adjustable constant such that $0 \leq a<1$. If $a=0, \eta_{i}=\Delta$; that is, the original uniform grid is recovered. As $a \rightarrow 1$, the clustering becomes extreme. The new grid points are

$$
Z_{1}=z_{B} ; \quad Z_{j}=z_{B}+\sum_{i=1}^{j-1} \eta_{i} ; \quad j=2, \ldots, N .
$$

\section{APPENDIX B}

\section{The Finite-Difference Method}

The most commonly used local method is the finitedifference (FD) method, since it is well suited to problems in complex geometries and boundary conditions. On regular grids $(a=0)$, the FD method is simple. The $(i, j)$ th component of the derivative matrix, $D_{i, j}^{(K)}$, is derived using Taylor series expansions and tabulated (e.g., Abramowitz and Stegun 1964; Bickley 1941). However, it becomes difficult to construct the differencing formula using Taylor's expansion on irregular grids, especially for high-degree and high-order derivatives. Instead, we use polynomial interpolation, which is suitable for calculating derivative matrices of any degree with any order of accuracy on an arbitrary grid. For barycentric Lagrange interpolation (Klein and Berrut 2012; Berrut and Klein 2014), we expand the function to be differentiated, $f(Z)$, as

$$
f\left(Z_{i}\right)=\frac{\sum_{j=j_{1}}^{j_{2}} \frac{\beta_{j}}{Z_{i}-Z_{j}} f\left(Z_{j}\right)}{\sum_{j=j_{1}}^{j_{2}} \frac{\beta_{j}}{Z_{i}-Z_{j}}}, \quad i=1,2, \ldots, N,
$$

where $j_{1}$ and $j_{2}$ are identify neighboring grid points around the derivative point $i$. For example, the fourthorder central finite-difference scheme for first derivatives involves the grid points from $j_{1}=i-2$ to $j_{2}=i+2$. The stencil width is $J=j_{2}-j_{1}+1$ ( 5 , in this case). When $J<N$, Eq. (B1) is a local method, whereas it is a global method when $J=N$. The values $\beta_{j}=\left[\prod_{i \neq j}\left(Z_{j}-Z_{i}\right)\right]^{-1}$ are called the barycentric weights. The $K$ th derivative of $f(Z)$ is then given by

$$
f^{(K)}\left(Z_{i}\right)=\sum_{j=j_{1}}^{j_{2}} D_{i, j}^{(K)} f\left(Z_{j}\right), \quad i=1,2, \ldots, N,
$$

where the differentiation matrix ${\underset{\sim}{\mathbf{D}}}^{(K)}$ has components

$$
\begin{aligned}
& D_{i, j}^{(1)}=\left\{\begin{array}{l}
\frac{\beta_{j}}{\beta_{i}} \frac{1}{Z_{i}-Z_{j}}, \quad i \neq j \\
-\sum_{\substack{m=1 \\
m \neq i}}^{N} D_{i, m}^{(1)}, \quad i=j
\end{array}\right. \\
& D_{i, j}^{(K)}= \begin{cases}\frac{K}{Z_{i}-Z_{j}}\left[\frac{\beta_{j}}{\beta_{i}} D_{i, i}^{(K-1)}-D_{i, j}^{(K-1)}\right], & i \neq j \\
-\sum_{\substack{m=1 \\
m \neq i}}^{N} D_{i, m}^{(K)}, \quad K \geq 2 .\end{cases}
\end{aligned}
$$

Boundary conditions are expressed via a matrix $\underset{\sim}{\mathbf{C}}$ and a vector $\mathbf{b}$ such that

$$
\underset{\sim}{\mathbf{C x}}=\mathbf{b},
$$

where $\mathbf{x}$ is the vector of eigenfunctions as defined in section 3 . If the total number of boundary conditions is $N_{\mathrm{bc}}$, then $\mathbf{C}$ is an $N_{\mathrm{bc}} \times 2 N$ matrix with each row specifying one boundary condition. Similarly, b is an $N_{\mathrm{bc}} \times 1$ column vector. For example, if the boundary condition requires that the first element of $\mathbf{x}$ vanish (a homogeneous Dirichlet condition), then the top row of $\underset{\sim}{\mathbf{C}}$ is $[1,0,0, \ldots]$ and the top value of $\mathbf{b}$ is 0 . If the boundary condition involves derivatives, then $\underset{\sim}{\mathbf{C}}$ will include elements of the appropriate derivative matrix.

Now, we decompose $\mathbf{x}$ into $\mathbf{x}_{k}$, the part of $\mathbf{x}$ that we will keep, and $\mathbf{x}_{r}$, the part that we will remove. The decomposition is chosen so that the boundary conditions become

$$
\left[\begin{array}{cc}
\mathbf{C}_{k} & 0 \\
0 & \mathbf{C}_{r}
\end{array}\right]\left[\begin{array}{c}
\mathbf{x}_{k} \\
\mathbf{x}_{r}
\end{array}\right]=\mathbf{b}
$$

Solving Eq. (B4), $\mathbf{x}_{r}$ can be expressed as a function of $\mathbf{x}_{k}$ :

$$
\mathbf{x}_{r}=\underbrace{-\mathbf{C}_{r}^{-1} \mathbf{C}_{k}}_{\underset{\sim}{\sim}} \mathbf{x}_{k}+\underbrace{\mathbf{C}_{r}^{-1}}_{\underset{\sim}{\sim}} \mathbf{b} .
$$

The matrix $\underset{\sim}{\mathbf{H}}$ allows for possible nonhomogeneous boundary conditions. In the present applications the boundary conditions are homogeneous $(\mathbf{b}=0)$ and Eq. (B5) is simply

$$
\mathbf{x}_{r}=\underset{\sim}{\mathbf{G}} \mathbf{x}_{k}
$$

The matrix $\mathbf{G}$ is called the "give-back" matrix, since it gives back the removed degrees of freedom. 
The eigenvalue Eq. (7) can likewise be separated into two parts:

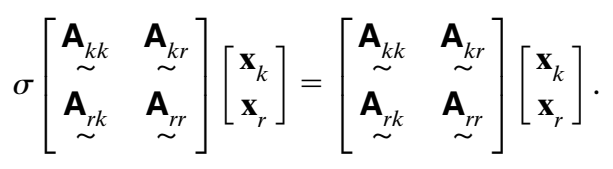

We are only interested in $\mathbf{x}_{k}$, since $\mathbf{x}_{r}$ can be recovered using Eq. (B6). Beginning with the upper part of Eq. (B7),

$$
\sigma\left(\mathbf{A}_{\sim k} \mathbf{x}_{k}+\underset{\sim}{\mathbf{A}_{k}} \mathbf{x}_{r}\right)=\underset{\sim}{\mathbf{B}_{k k} \mathbf{x}_{k}}+\underset{\sim}{\mathbf{B}_{k r}} \mathbf{x}_{r},
$$

we substitute Eq. (B6), obtaining

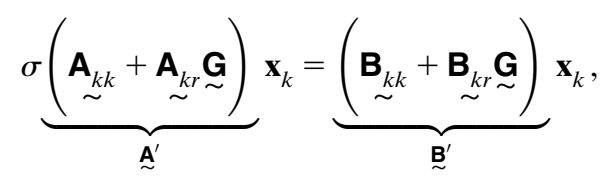

where $\underset{\sim}{\mathbf{A}^{\prime}}$ and $\underset{\sim}{\mathbf{B}^{\prime}}$ are matrices modified to account for the boundary conditions. The generalized eigenvalue to be solved is then

$$
\begin{aligned}
& \sigma \underset{\sim}{\mathbf{A}^{\prime}} k={\underset{\sim}{\mathbf{B}^{\prime}}}^{\prime} \mathbf{x}_{k} . \\
& C_{i, j}^{(1)}=\left\{\begin{array}{ll}
\frac{b_{j}}{b_{i}} \frac{(-1)^{i+j}}{c_{i}-c_{j}}, & i \neq j \\
-\sum_{\substack{m=1 \\
m \neq i}} D_{i, m}^{(1)}, & i=j
\end{array}, \quad C_{i, j}^{(K)}=\left\{\begin{array}{ll}
\frac{K}{c_{i}-c_{j}}\left[\frac{b_{j}(-1)^{i+j}}{b_{i}} D_{i, i}^{(K-1)}-D_{i, j}^{(K-1)}\right], & i \neq j \\
-\sum_{\substack{m=1 \\
m \neq i}} D_{i, m}^{(K)}, & i=j
\end{array} \quad K \geq 2,\right.\right.
\end{aligned}
$$

After $\sigma$ and $\mathbf{x}_{k}$ are obtained from Eq. (B8), we can recover $\mathbf{x}_{r}$ using Eq. (B6), and finally assemble the eigenvector $\mathbf{x}$

\section{APPENDIX C}

\section{The Chebyshev-Collocation Method}

Polynomial (including the Chebyshev polynomials) interpolation on equally spaced points is known to suffer from the Runge phenomenon (Fornberg and Zuev 2007). The best way to overcome this problem is to use irregular distributed interpolation points, such as the most common and simplest one, the Chebyshev-Gauss-Lobatto points,

$$
c_{i}=\cos \left(\frac{i-1}{N-1} \pi\right), \quad i=1, \ldots, N,
$$

which is the extrema of Chebyshev polynomials $T_{i}(c)=$ $\cos \left[i \cos ^{-1}(c)\right]$. We can visualize these points as the projections on $[-1,1]$ of equispaced points $z_{i}$ on the upper half of the unit circle (see Fig. 5.1 of Trefethen 2000). They are clustered at boundaries and should be very effective to analyze the boundary layer instability. Substituting Eq. (C1) into Eq. (B3), we get the where $b_{j}=1 / 2$ if $j=1$ or $N$, and $b_{j}=1$ otherwise. The Chebyshev points and matrices are defined on $[-1,1]$; they can be extended to $\left[Z_{B}, Z_{T}\right]$ by using $Z_{i}=Z_{B}+$ $\left[\left(Z_{T}-Z_{B}\right) / 2\right]\left(c_{i}+1\right)$ and $\mathbf{D}^{(K)}=\mathbf{C}^{(K)} /\left[\left(Z_{T}-Z_{B}\right) / 2\right]^{K}$.

We find that the collocation method is nothing other than the barycentric Lagrange interpolation based on the Chebyshev collocation points. The boundary conditions can be incorporated using the same approach discussed in the last section.

\section{APPENDIX D}

\section{The Fourier-Galerkin Method}

Here we describe a Fourier-Galerkin method in which the solution is expanded in terms of sine functions:

$$
F_{n}(Z)=\sqrt{\frac{2}{H}} \sin \frac{n \pi}{H} Z
$$

Then

$$
\hat{w}(Z)=\sum_{n=1}^{N} w_{n} F_{n}(Z), \quad \hat{b}(Z)=\sum_{n=1}^{N} b_{n} F_{n}(Z) .
$$

Here, $F_{n}(Z)$ automatically satisfies the frictionless boundary conditions $\hat{x}=\hat{x}^{\prime \prime}=0$ at $Z=0$ and $Z=H$. This choice of basis functions is natural for impermeable, frictionless, constant-buoyancy boundaries, and may be adapted for insulating boundaries by expanding $\hat{b}$ in terms of cosines rather than sines. But the method is not well-suited for rigid boundaries.

The orthogonality of $F_{n}(Z)$ is expressed by 


$$
\int_{0}^{H} F_{m}(Z) F_{n}(Z) d Z=\boldsymbol{\delta}_{m, n},
$$

where $\boldsymbol{\delta}_{m, n}$ is the $N \times N$ identity matrix. Substituting Eq. (D1) into Eqs. (1) and (2) gives

$$
\begin{gathered}
(\sigma+i k U) \sum_{n=1}^{N} w_{n} \nabla^{2} F_{n}-i k U^{\prime \prime} \sum_{n=1}^{N} w_{n} F_{n} \\
=T_{w} \sum_{n=1}^{N} w_{n} F_{n}-\tilde{k}^{2} \sum_{n=1}^{N} b_{n} F_{n},
\end{gathered}
$$

$$
(\sigma+i k U) \sum_{n=1}^{N} b_{n} F_{n}+B^{\prime} \sum_{n=1}^{N} w_{n} F_{n}=T_{b} \sum_{n=1}^{N} b_{n} F_{n},
$$

where primes indicate differentiation with respect to $Z$. Using the fact that $F_{n}^{\prime \prime}=-(n \pi / H)^{2} F_{n}$, the Laplacian $\nabla^{2} F_{n}$ becomes $D_{n} F_{n}$, where

$$
D_{n}=-\left(\frac{n \pi}{H}\right)^{2}-\tilde{k}^{2} .
$$

The viscous terms $T_{w} F_{n}$ and $T_{b} F_{n}$ become

$$
\begin{aligned}
T_{w} F_{n}= & \frac{d^{2} A_{v}}{d Z^{2}}\left[-\left(\frac{n \pi}{H}\right)^{2}\right] F_{n}+2 \frac{d A_{v}}{d Z}\left[-\left(\frac{n \pi}{H}\right)^{3}\right] \underbrace{\sqrt{\frac{2}{H}} \cos \frac{n \pi}{H} Z}_{G_{n}}+A_{v}\left(\frac{n \pi}{H}\right)^{4} F_{n} \\
& -\tilde{k}^{2} \frac{d\left(A_{h}+A_{v}\right)}{d Z}\left(\frac{n \pi}{H}\right) \underbrace{\sqrt{\frac{2}{H}} \cos \frac{n \pi}{H} Z}_{G_{n}}-\tilde{k}^{2}\left(A_{h}+A_{v}\right)\left[-\left(\frac{n \pi}{H}\right)^{2}\right] F_{n}+\tilde{k}^{4} A_{h} F_{n}, \\
T_{b}= & \frac{d K_{v}}{d Z}\left(\frac{n \pi}{H}\right) \underbrace{\sqrt{\frac{2}{H}} \cos \frac{n \pi}{H} Z}_{G_{n}}+K_{v}\left[-\left(\frac{n \pi}{H}\right)^{2}\right] F_{n}-\tilde{k}^{2} K_{h} F_{n} .
\end{aligned}
$$

Now multiply Eqs. (D3) and (D4) by $F_{m}$ and integrate,

remembering Eq. (D2):

$$
\begin{aligned}
& \sigma \sum_{n=1}^{N} w_{n} D_{n} \underbrace{\int_{0}^{H} F_{m} F_{n} d Z}_{=\delta_{m, n}}=-i k \sum_{n=1}^{N} w_{n} D_{n} \int_{0}^{H} F_{m} U F_{n} d Z+i k \sum_{n=1}^{N} w_{n} \int_{0}^{H} F_{m} U^{\prime \prime} F_{n} d Z \\
& -k^{2} \sum_{n=1}^{N} b_{n} \underbrace{\int_{0}^{H} F_{m} F_{n} d Z}_{=\delta_{m, n}}+\sum_{n=1}^{N} w_{n}\left[-\left(\frac{n \pi}{H}\right)^{2}\right] \int_{0}^{H} F_{m} A_{v}^{\prime \prime} F_{n} d Z+2 \sum_{n=1}^{N} w_{n}\left[-\left(\frac{n \pi}{H}\right)^{3}\right] \int_{0}^{H} F_{m} A_{v}^{\prime} G_{n} d Z \\
& +\sum_{n=1}^{N} w_{n}\left(\frac{n \pi}{H}\right)^{4} \int_{0}^{H} F_{m} A_{v} F_{n} d Z-k^{2} \sum_{n=1}^{N} w_{n} \frac{n \pi}{H} \int_{0}^{H} F_{m}\left(A_{h}+A_{v}\right)^{\prime} G_{n} d Z \\
& -k^{2} \sum_{n=1}^{N} w_{n}\left[-\left(\frac{n \pi}{H}\right)^{2}\right] \int_{0}^{H} F_{m}\left(A_{h}+A_{v}\right) F_{n} d Z+k^{4} \sum_{n=1}^{N} w_{n} \int_{0}^{H} F_{m} A_{h} F_{n} d Z, \\
& \sigma \sum_{n=1}^{N} b_{n} \underbrace{\int_{0}^{H} F_{m} F_{n} d Z}_{=\delta_{m, n}}=-i k \sum_{n=1}^{N} b_{n} \int_{0}^{H} F_{m} U F_{n} d Z-\sum_{n=1}^{N} w_{n} \int_{0}^{H} F_{m} B^{\prime} F_{n} d Z+\sum_{n=1}^{N} b_{n} \frac{n \pi}{H} \int_{0}^{H} F_{m} K_{v}^{\prime} G_{n} d Z \\
& +\sum_{n=1}^{N} b_{n}\left[-\left(\frac{n \pi}{H}\right)^{2}\right] \int_{0}^{H} F_{m} K_{v} F_{n} d Z-\tilde{k}^{2} \sum_{n=1}^{N} b_{n} \int_{0}^{H} F_{m} K_{h} F_{n} d Z .
\end{aligned}
$$


The summation on the left-hand sides of Eqs. (D5) and (D6) can be done explicitly, resulting in $w_{m} D_{m}$ and $b_{m}$, respectively. Then Eqs. (D5) and (D6) now become the algebraic eigenvalue problem;

$$
\sigma\left[\begin{array}{c}
w_{m} \\
b_{m}
\end{array}\right]=\left[\begin{array}{cc}
B_{11} & -\tilde{k}^{2} \frac{1}{D_{m}} \\
-\int_{0}^{H} F_{m} B^{\prime} F_{n} d Z & B_{22}
\end{array}\right]\left[\begin{array}{l}
w_{m} \\
b_{m}
\end{array}\right],
$$

$$
\begin{aligned}
B_{11}= & -i k \frac{D_{n}}{D_{m}} \int_{0}^{H} F_{m} U F_{n} d Z+i k \frac{1}{D_{m}} \int_{0}^{H} F_{m} U^{\prime \prime} F_{n} d Z-\frac{(n \pi / H)^{2}}{D_{m}} \int_{0}^{H} F_{m} A_{v}^{\prime \prime} F_{n} d Z \\
& -2 \frac{(n \pi / H)^{3}}{D_{m}} \int_{0}^{H} F_{m} A_{v}^{\prime} G_{n} d Z+\frac{(n \pi / H)^{4}}{D_{m}} \int_{0}^{H} F_{m} A_{v} F_{n} d Z-\frac{\tilde{k}^{2}(n \pi / H)}{D_{m}} \int_{0}^{H} F_{m}\left(A_{h}+A_{v}\right)^{\prime} G_{n} d Z \\
& +\frac{\tilde{k}^{2}(n \pi / H)^{2}}{D_{m}} \int_{0}^{H} F_{m}\left(A_{h}+A_{v}\right) F_{n} d Z+\frac{\tilde{k}^{4}}{D_{m}} \int_{0}^{H} F_{m} A_{h} F_{n} d Z,
\end{aligned}
$$

$$
\begin{aligned}
B_{22}= & -i k \int_{0}^{H} F_{m} U F_{n} d Z+\frac{n \pi}{H} \int_{0}^{H} F_{m} K_{v}^{\prime} G_{n} d Z \\
& -\left(\frac{n \pi}{H}\right)^{2} \int_{0}^{H} F_{m} K_{v} F_{n} d Z-\tilde{k}^{2} \int_{0}^{H} F_{m} K_{h} F_{n} d Z .
\end{aligned}
$$

The eigenvalue is the growth rate $\sigma$ as usual, but the eigenvector is now composed of the coefficients $w_{n}$ and $b_{n}$ in the expansion (D1).

\section{APPENDIX E}

\section{List of Symbols}

k Wave vector

$k \quad$ Wave vector magnitude

$U$ Background flow parallel to the wave vector

$B_{z} \quad$ Squared buoyancy frequency

$\rho \quad$ Density

$\rho_{0} \quad$ Characteristic value of density

$\bar{\rho} \quad$ Mean density

$\rho^{\prime} \quad$ Density perturbation

$b \quad$ Buoyancy

$b^{\prime} \quad$ Buoyancy perturbation

$w^{\prime} \quad$ Vertical velocity perturbation

$\hat{w} \quad$ Complex vertical structure function of vertical velocity

$\hat{b} \quad$ Complex vertical structure function of buoyancy

$\sigma \quad$ Exponential growth rate of instability

$\mathrm{Ri}_{b} \quad$ Bulk Richardson number

Re Reynolds number

\section{REFERENCES}

Abramowitz, M., and I. A. Stegun, 1964: Handbook of Mathematical Functions with Formulas, Graphs, and Mathematical Tables. Vol. 55. U.S. Government Printing Office, 1046 pp.

Berrut, J.-P., and G. Klein, 2014: Recent advances in linear barycentric rational interpolation. J. Comput. Appl. Math., 259, 95-107, https://doi.org/10.1016/j.cam.2013.03.044.

Bickley, W., 1941: Formulae for numerical differentiation. Math. Gaz., 25, 19-27, https://doi.org/10.1017/S002555720007008X.

Fjørtoft, R., 1950: Application of integral theorems in deriving criteria of stability for laminar flows and for the baroclinic circular vortex. Geofys. Publ., 17 (6), 1-52.

Fornberg, B., and J. Zuev, 2007: The Runge phenomenon and spatially variable shape parameters in RBF interpolation. Comput. Math. Appl., 54, 379-398, https://doi.org/10.1016/ j.camwa.2007.01.028.

Goldstein, S., 1931: On the stability of superposed streams of fluids of different densities. Proc. Roy. Soc. London, 132A, 524-548, https://doi.org/10.1098/rspa.1931.0116.

Gregg, M., E. D’Asaro, J. Riley, and E. Kunze, 2018: Mixing efficiency in the ocean. Annu. Rev. Mar. Sci., 10, 443-473, https:// doi.org/10.1146/annurev-marine-121916-063643.

Hazel, P., 1972: Numerical studies of the stability of inviscid stratified shear flows. J. Fluid Mech., 51, 39-61, https://doi.org/ 10.1017/S0022112072001065.

Howard, L. N., 1961: Note on a paper of John W. Miles. J. Fluid Mech., 10, 509-512, https://doi.org/10.1017/S0022112061000317.

Klein, G., and J.-P. Berrut, 2012: Linear rational finite differences from derivatives of barycentric rational interpolants. SIAM J. Numer. Anal., 50, 643-656, https://doi.org/10.1137/ 110827156.

Liu, Z., 2010: Instability of baroclinic tidal flow in a stratified fjord. J. Phys. Oceanogr., 40, 139-154, https://doi.org/ 10.1175/2009JPO4154.1. 
S. A. Thorpe, and W. D. Smyth, 2012: Instability and hydraulics of turbulent stratified shear flows. J. Fluid Mech., 695, 235-256, https://doi.org/10.1017/jfm.2012.13.

Maslowe, S. A., 1986: Critical layers in shear flows. Annu. Rev. Fluid Mech., 18, 405-432, https://doi.org/10.1146/ annurev.fl.18.010186.002201.

Miles, J. W., 1961: On the stability of heterogeneous shear flows. J. Fluid Mech., 10, 496-508, https://doi.org/10.1017/ S0022112061000305.

Putrevu, U., and I. A. Svendsen, 1992: Shear instability of longshore currents: A numerical study. J. Geophys. Res., 97, 72837303, https://doi.org/10.1029/91JC02988.

Rayleigh, L., 1880: On the stability or instability of certain fluid motions. Scientific Papers, Vol. 3, Cambridge University Press, 594-596.

Rees, T., and A. Monahan, 2014: A general numerical method for analyzing the linear stability of stratified parallel shear flows. J. Atmos. Oceanic Technol., 31, 2795-2808, https://doi.org/ 10.1175/JTECH-D-14-00034.1.

Schlichting, H., and K. Gersten, 2016: Boundary-Layer Theory. Springer, $805 \mathrm{pp}$.

Smyth, W. D., and W. R. Peltier, 1989: The transition between Kelvin-Helmholtz and Holmboe instability: An investigation of the overreflection hypothesis. J. Atmos. Sci., 46, 3698-3720, https://doi.org/10.1175/1520-0469(1989)046<3698:TTBKAH> 2.0.CO;2.

— - and J. R. Carpenter, 2019: Instability in Geophysical Flows. Cambridge University Press, 338 pp.

- J. N. Moum, and J. D. Nash, 2011: Narrowband oscillations in the upper equatorial ocean. Part II: Properties of shear instabilities. J. Phys. Oceanogr., 41, 412-428, https://doi.org/ 10.1175/2010JPO4451.1.

,,-- L. Li, and S. A. Thorpe, 2013: Diurnal shear instability, the descent of the surface shear layer, and the deep cycle of equatorial turbulence. J. Phys. Oceanogr., 43, 2432-2455, https://doi.org/10.1175/JPO-D-13-089.1.

Sun, C., W. Smyth, and J. Moum, 1998: Dynamic instability of stratified shear flow in the upper equatorial Pacific. J. Geophys. Res., 103, $10323-10337$, https://doi.org/10.1029/ 98JC00191.

Taylor, G. I., 1931: Effect of variation in density on the stability of superposed streams of fluid. Proc. Roy. Soc. London, 132A, 499-523, https://doi.org/10.1098/rspa.1931.0115.

Trefethen, L. N., 2000: Spectral Methods in MATLAB. Society for Industrial and Applied Mathematics, $165 \mathrm{pp}$. 